\title{
The enriched chromium neutrino source for GALLEX
}

18 Jan 1991

Brookhaven National Lab., Upton, NY (USA)

Reproduced and Distributed by:

\section{U.S. DEPARTMENT OF ENERGY}

Office of Scientific and Technical Information

P.O. Box 62

Oak Ridge, TN 37831 


\title{
THE ENRICHED CHROMIUM NEUTRINO SOURCE FOR GALLEX*
}

\author{
F.X. Hartmann and R.L. Hahn**
}

January 18, 1991

*This research was carried out at Brookhaven National Laboratory under contract DE-ACO2-76CHOOO16 with the U.S. Department of Energy and supported by its Office of High Energy and Nuclear Physics.

**The members of the Solar Neutrino Group in the BNL Chemistry Department are participating in the International GALLEX Collaboration. 


\section{DISCLAIMER}

This report was prepared as an account of work sponsored by an agency of the United States Government. Neither the United States Governmeni nor any agency thereof. nor any of their employees, nor any of their contractors, subcontractors. or their employees, makes any warranty, express or implied. or assumes any legal liability or responsibility for the accuracy, completeness, or usefulness of any information. apparatus, product, or process disclosed, or represents that its use would not infringe privately owned rights. Reference herein to any specific commercial product. process. or service by trade name, trademark, manufacturer, or otherwise, does not necesgarily constitute or imply its endorsement, recommendation. or favoring by the United States Government or any agency, contractor or subcontractor thereof. The views and opinions of authors expressed herein do not necessarily state or reflect those of the United States Government or any agency. contractor or subcontractor thereof.

Printed in the United Stateg of America

Available from

National Technical Information Service

U.S. Department of Commerce

5285 Port Royal Road

Springfield, VA 22161

NTIS price codes:

Printed Copy: A04; Microfiche Copy: A01 
Abstract. The preparation and study of an intense source of neutrinos in the form of neutron irradiated materials which are enriched in Cr-50 for use in the GALLEX solar neutrino experiment are discussed. Chromyl fluoride gas is enriched in the $\mathrm{Cr}-50$ isotope by gas centrifugation and subsequently converted to a very stable form of chromium oxide. The results of neutron activation analyses of such chromium samples indicate low levels of any long-lived activities, but show that short-lived activities, in particular Na-24, may be of concern. These results show that irradiating chromium oxide enriched in $\mathrm{Cr}-50$ is preferable to irradiating either natural chromium or argon gas as a means of producing a neutrino source to calibrate the GALLEX detector. These results of the impurity level analysis of the enriched chromyl fluoride gas and its conversion to the oxide are also of interest to work in progress by other members of the Collaboration investigating an alternative conversion of the enriched gas to chromium metal. 


\section{Contents}

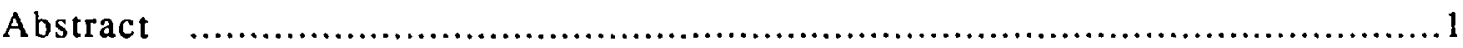

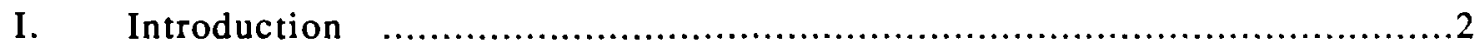

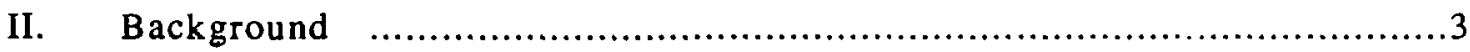

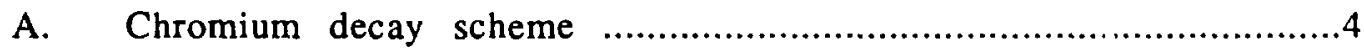

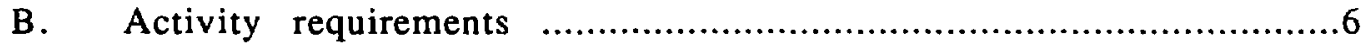

C. Source design considerations …...............................................

III. Enriched Chromium Source Preparations and Test Irradiations ..............................10

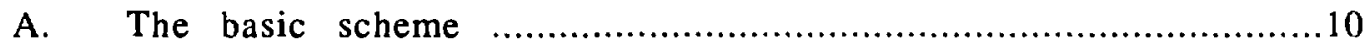

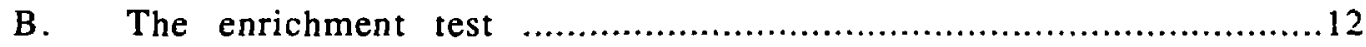

C. Conversion to enriched chromium oxide ...........................................16

D. Apparatus to handle chromyl fluoride ..............................................

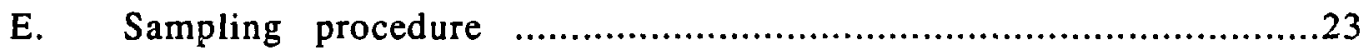

F. Reduction and firing of $\mathrm{Cr}_{2} \mathrm{O}_{3} \Sigma \mathrm{XH}_{2} \mathrm{O}$ to produce high purity $\mathrm{Cr}_{2} \mathrm{O}_{3} \ldots \ldots \ldots \ldots . . .25$

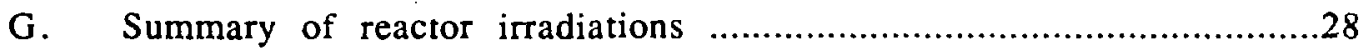

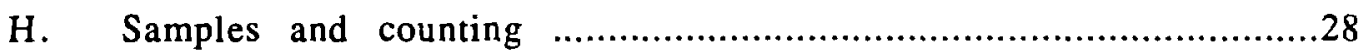

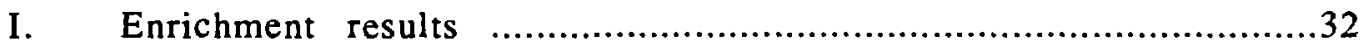

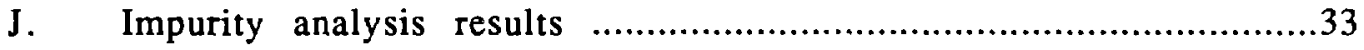

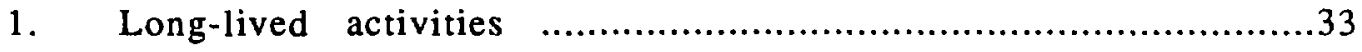

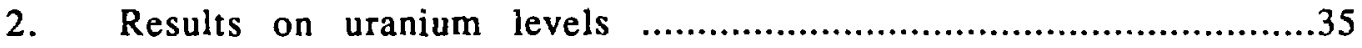

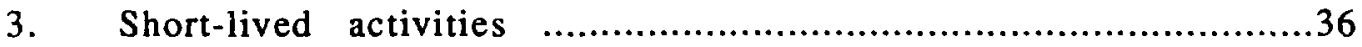

4. Possible sources of impurities .........................................40

IV. Expected Radiation Rates From the 1-MCi Source ..................................44

V. Summary of Radiation Levels from Chromium Tests ..........................................47

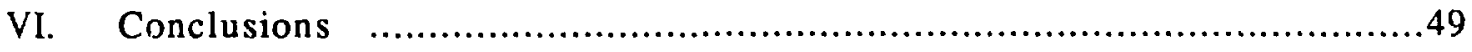

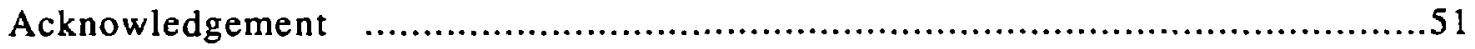

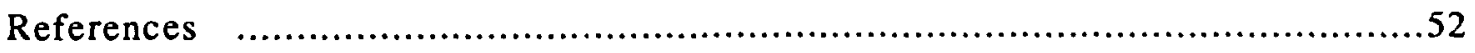




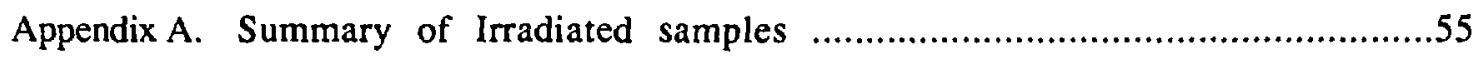

Appendix B. Nuclear Data Relevant to Chromium Impurity Analyses .............................57

Appendix C. Complete Listing of Analyzed Impurities in $\mathrm{Cr}_{2} \mathrm{O}_{3}$ Samples ……................60

Appendix D. Summary of Hazardous Impurities and Radiation Rate Data ..........................62 


\section{Introduction.}

The experimental measurements of the solar neutrino flux by the Homestake Chlorine Detector ${ }^{1}$ and by the Kamiokande II water Cherenkov detector $^{2}$ give results that significantly differ from those which are predicted from solar models. ${ }^{3,4}$ Specifically, the Homestake Chlorine Detector result of $2.33 \pm 0.25$ SNU is $40 \pm 11 \%$ of the solar neutrino flux predicted by the Turck-Chieze Solar Model and $29 \pm 11 \%$ of the flux predicted by the Standard Solar Model (SSM); the Kamiokande II detector results are $46 \pm 17 \%$ of the neutrino flux which is predicted by the SSM. 2,5 The two gallium experiments, GALLEX 5 and SAGE ${ }^{6}$, which are sensitive in the energy regime corresponding to the $p-p$ solar neutrino flux, are specifically designed to help resolve this discrepancy, often referred to as the "solar neutrino problem".

The gallium detector results, though, may well serve to provide constraints on the fundamental properties of the neutrinos. As calculated by Baltz and Weneser ${ }^{7}$ using the theory developed by Mikheyev, Smirnov 8 and Wolfenstein ${ }^{9}$ (MSW), a wide range of values from 0 to $100 \%$ of the SSM prediction are possible for the gallium detector if the free neutrino is not a weak-interaction eigenstate and at least one neutrino flavor has mass. ${ }^{10}$ The implications of an experimental result which is lower than the SSM prediction of 132 SNU for the gallium experiments, for example, were emphasized in a recent prediction by Bahcall and Bethe ${ }^{11}$ of 4.5 SNU (approximately plus or minus a factor of 2 ) for the gallium detector, based on the MSW effect and analysis of the Chlorine and Kamiokande-II detector results.

The experimental plans in both gallium experiments include the use of a test source of neutrinos to check the operation of the detectors. Such a check would provide direct and convincing evidence that all parts of the detector work 
correctly in response to a real neutrino interaction in the gallium. This is particularly important since the gallium detectors are radiochemical detectors; the resulting germanium ion is not a noble gas atom (such as argon in the Homestake Chlorine Detector) and thus potential chemical side reactions could occur. Moreover the recently announced low result from the SAGE experiment ${ }^{6}$ enhances the need for the full calibration of the detector system.

Calibration sources based on chromium-51 are currently under active consideration. The approach currently being pursued in SAGE is to irradiate highly enriched (87.7\%) Cr-50 in a metallic form. ${ }^{12}$ The GALLEX Collaboration plans to use partially enriched Cr-50 (about 20-40\%) in either the metallic or the oxide form.

This report summarizes our studies of the production of $\mathrm{Cr}-51$ in neutron activations of $\mathrm{Cr}-50$ enriched by gas centrifugation and prepared in the form of the oxide. Previous tests involved irradiation of natural chromium in the form of purchased metallic grains, as summarized in Ref. 13. In this work, we use the gas enrichment process, whereas SAGE uses electromagnetic separation. Parallel work is also currently underway by the GALLEX Collaboration involving enriched chromium converted to the metal by electrolysis. ${ }^{14}$ Clearly, both gallium collaborations believe that the $\mathrm{Cr}-51$ source is preferable to the Ar-37 source proposed by Haxton in a recent communication. 15

\section{Background.}

The use of radioactive $\mathrm{Cr}-51$ for the purposes of calibrating the gallium detector was first proposed by Raghavan. ${ }^{16}$ A Zn-65 source proposed by Alvarez ${ }^{17}$ has a much longer half life and emits higher energy gamma rays than the Cr-51, and so would require thicker shielding. ${ }^{12}$ 


\section{A. Chromium decay scheme.}

The nucleus $\mathrm{Cr}-51$ decays by electron capture to the $7 / 2$ - ground state and the first excited state (5/2-) of the V-51 nucleus with a half-life of 27.70 days, as depicted in Fig. 1. The first excited state of V-51 decays rapidly by the emission of a $320 \mathrm{KeV}$ gamma-ray; the ground state is stable. With a $Q$ value of $751.3 \mathrm{KeV}$, this source produces monoenergetic neutrinos with energies of $746,751,426$ and $431 \mathrm{KeV} .13$

The V-51 daughter atom de-excites by the emission of low-energy x-rays and Auger electrons, all of which are easily attenuated by shielding. The intensity of the $320 \mathrm{KeV}$ gamma-ray which is emitted in $10 \%$ of the decays can be easily reduced with relatively small amounts of shielding ( $\mathrm{few} \mathrm{cm}$ of lead). As discussed in Section IV below, though, it is those impurities at parts per million (ppm) levels which emit hard gamma rays (generally in the 1 to $2 \mathrm{MeV}$ range) that pose a serious radiation hazard.

In Fig. 1 we depict, as well, the four prominent neutrino lines from the decay of $\mathrm{Cr}-51$. The most prominent emission at $745.8 \mathrm{KeV}$ occurs $81 \%$ of the time, and the associated $750.7 \mathrm{KeV}$ line, $9 \%$. The $425.7 \mathrm{KeV}$ and $430.6 \mathrm{KeV}$ neutrinos have respective branching ratios of $9 \%$ and $1 \%$; all remaining neutrino emissions from $\mathrm{M}$ or valence $(\mathrm{V})$ shells occur less than $1 \%$ of the time. ${ }^{13}$

A number of nuclear states of Ge-71 can be populated in the neutrino absorption reaction:

$$
v+\mathrm{Ga}-71 \rightarrow \mathrm{Ge}-71^{*}+\mathrm{e}^{*}
$$

as depicted in the right side of Fig. 1. The $Q$ value for Ge-71 electron-capture decay is $233.2 \mathrm{KeV} .18$ The inverse beta-decay allows the population of three excited nuclear states in Ge-71, in addition to the ground state. Two of these three states, the $5 / 2^{-}$and $3 / 2^{-}$levels in Ge-71, can be populated by allowed Gamow-Teller transitions. 

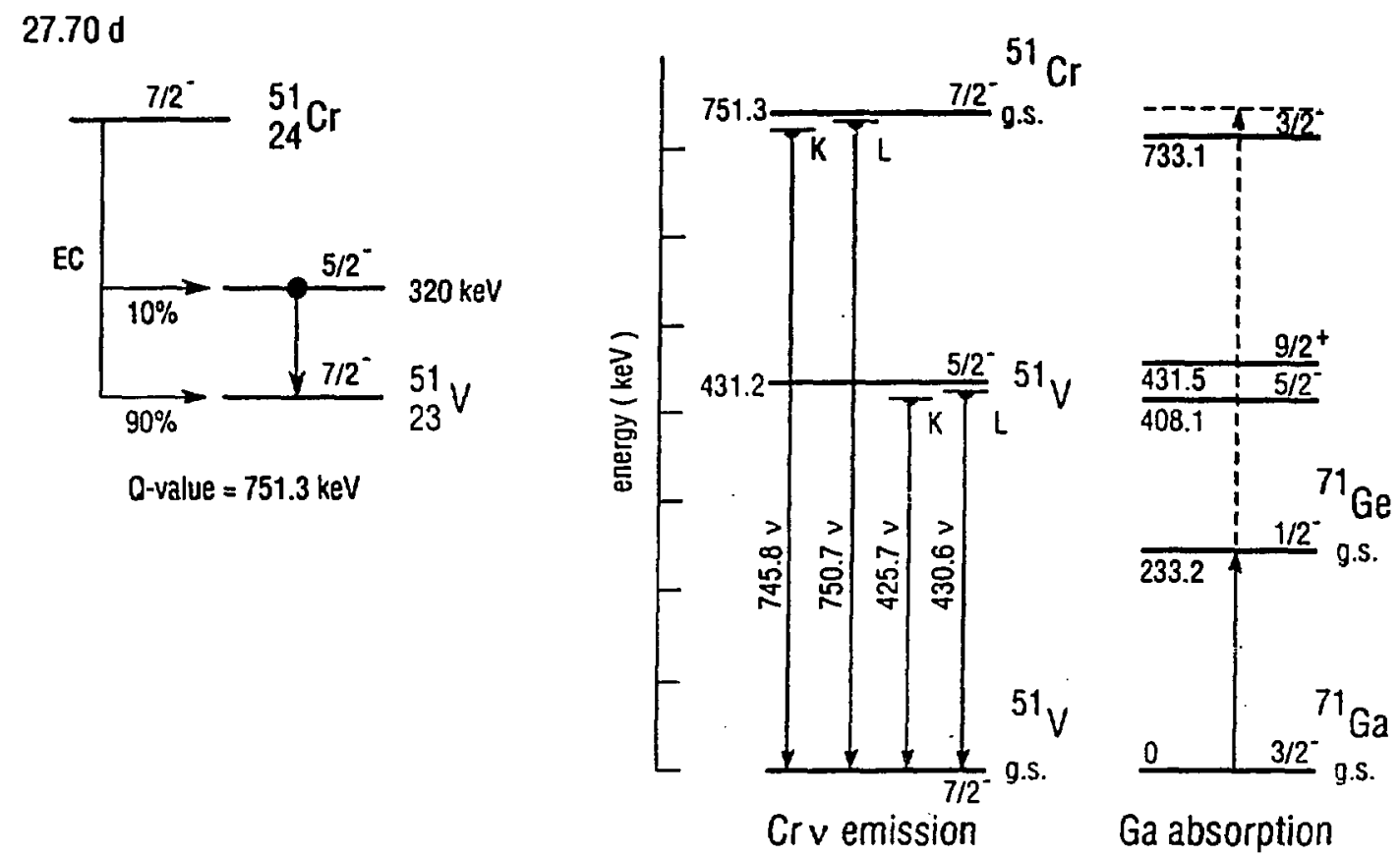

$B E(K$ in $V)=5.5 \mathrm{keV}$
$B E(L$ in $V)=0.6 \mathrm{keV}$
$E C(K) / E C(L)=10$

FIG. 1 Electron capture decay of Cr-51. The nucleus Cr-51 decays by electron capture to V-51 with a half-life of 27.7 days and the emission of low-energy $320-\mathrm{KeV}$ gamma rays. The decay emits neutrinos with essentially four discrete energies. - There neutrinos, as depicted in the right side of the figure,

energetically can convert Ga-71 into ground-state Ge-71, and excited Ge-71 states as indicated by the dashed line. All Ge-71 states depicted are very short-lived with the exception of the 11.43 day half-life ground state. The production rate of Ge-71 is then detected in the test. 
A description of the Ge-71 excited-state contributions to the cross-section for capture of Cr-51 neutrinos is discussed by Hampel in Ref. 19. The total Ge-71 electron-capture half-life of $11.43 \pm 0.03$ days measurea by Hampel and Remsberg ${ }^{20}$ sets the $\mathrm{ft}$ value at $2.179 \times 10^{4}$ seconds. From this value, the Ga-71 neutrino-absorption cross-section to populate the Ge-71 ground state can be determined. To obtain the contributions of the excited states, it is necessary to use the results obtained by Krofcheck et $a .^{21}$ at the Indiana University Cyclotron Facility. They studied experimentally the differential scattering cross-section in the (p,n) reaction on Ga-71 at proton energies of 125 and $200 \mathrm{MeV}$. The $(p, n)$ reaction mimics the neutrino-absorption reaction and can be used to determine the contrit: cross-section. Their measurements yield the ratio of the total cross-section to the ground-state cross-section to be 1.06 for $746 \mathrm{KeV} \mathrm{Cr}-51$ neutrinos and 1.02 for $426 \mathrm{KeV}$ neutrinos. The excited state contributions as determined by the Indiana University group are preferred over the first reported measurements by Orihara et al. ${ }^{22}$ at $35 \mathrm{MeV}$ since the lower energy $(p, n)$ results are not directly proportional to the Gamow-Teller transition strength.23

\section{B. Activity requirements.}

The activity requirements in the $\mathrm{Cr}$ calibration are discussed both by Hampel ${ }^{19}$ and by Cribier et al. 13 Many parameters enter into such activity estimates, but a convenient set of parameters assumes a total counter background of 0.12 counts per day in the acceptable (energy and rise-time) windows and an assumed solar neutrino production rate of $132 \mathrm{SNU}$. The results of such a study indicate that an enriched source of chromium, irradiated for 21 days in the Siloé nuclear reactor in France, will have a $1.08 \mathrm{MCi}$ initial decay rate. Such a source, used twice, gives the desired $10 \%$ calibration of the experiment. 
production of $\mathrm{Cr}-51$

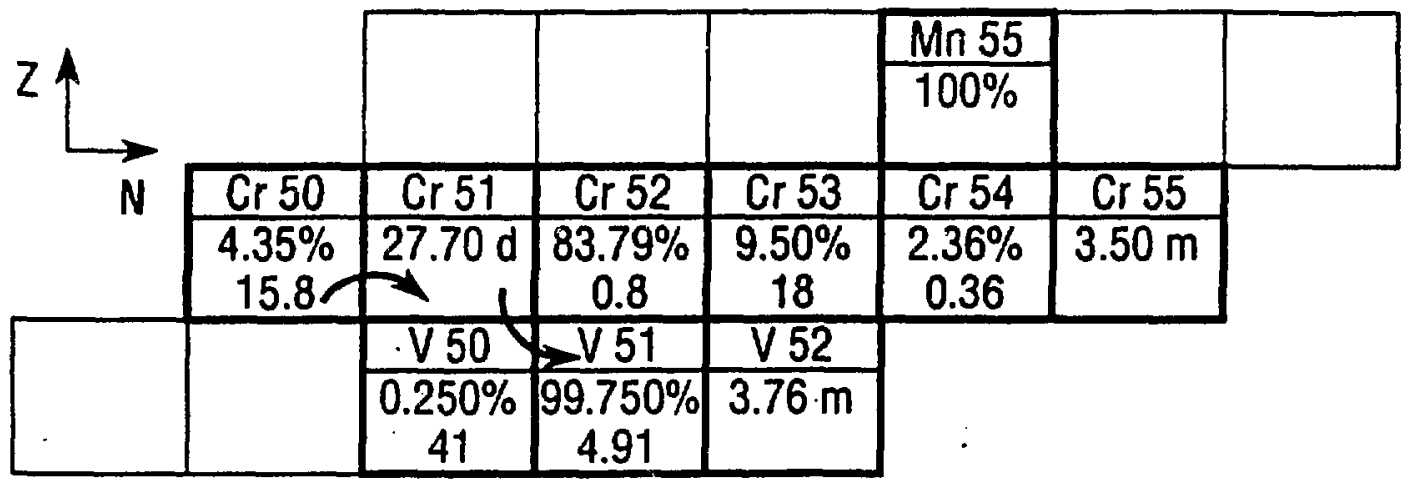

FIG. 2 Neutron production of $\mathrm{Cr}-51$. The nuclides relevant to the neutron irradiation of chromium are summarized along with their natural isotopic abundances or half-lives and thermal-neutron absorption cross-sections. Neutron activation of in-grown V-51 leads to stable Cr-52, and likewise Cr-54 gives stable Mn-55. Thus the $320 \mathrm{KeV}$ gamma ray from $\mathrm{Cr}-51$ is the only long-lived emission in pure chromium material. 
A source of natural chromium, irradiated for a comparable length of time and also used twice, at best gives an $18 \%$ calibration of the detector.

Radioactive $\mathrm{Cr}-51$ is produced by thermal neutron absorption of $\mathrm{Cr}-50$. The isotopic abundances of the chromium isotopes along with their thermal-neutren absorption cross-sections are depicted in Fig. 2.

The 3.50 minute $\mathrm{Cr}-55$, which is the only other radioactive $\mathrm{Cr}$ isotope produced in the thermal irradiation of chromium, decays to stable Mn-55. The stable V-51, formed from decay of the Cr-51, is the predominant daughter nucleus produced in the irradiation. Subsequent neutron capture produces V-52 which then decays with a 3.76 minute half-life to stable Cr-52.

\section{Source design considerations.}

The calibration requirements presented by Cribier $^{13}$ and the need for the enriched source can be conveniently and simply summarized by considering the reactor irradiation summary depicted in Fig. 3. (This plot is included as introductory material and not intended to address the numerous important details under study by the GALLEX group at Grenoble and Saclay.) There we plot the Cr-51 activity levels produced as functions of the thermal-neutron flux of the reactor and the irradiation time. The enrichment factor $\eta$ is the percentage of $\mathrm{Cr}-50$ in the source divided by the natural $\mathrm{Cr}-50$ isotopic composition (4.35\%). The picture clearly shows the basic items under consideration.

We consider the case of the $1.08 \mathrm{MCi}$ source. Such a source can be produced ideally in a 21-day reactor irradiation (typical of the reactor irradiation interval) from a natural chromium target $(\eta=1)$ at a total flux of $27 \times 10^{13}$ neutrons $/ \mathrm{cm}^{2}$-sec. The effective thermal neutron flux that was seen in the important natural-chromium test is $7 \times 10^{13}$ neutrons $/ \mathrm{cm}^{2}$-sec; thus four such "reactors" are needed to produce the total initial activity. If the number of reactor 


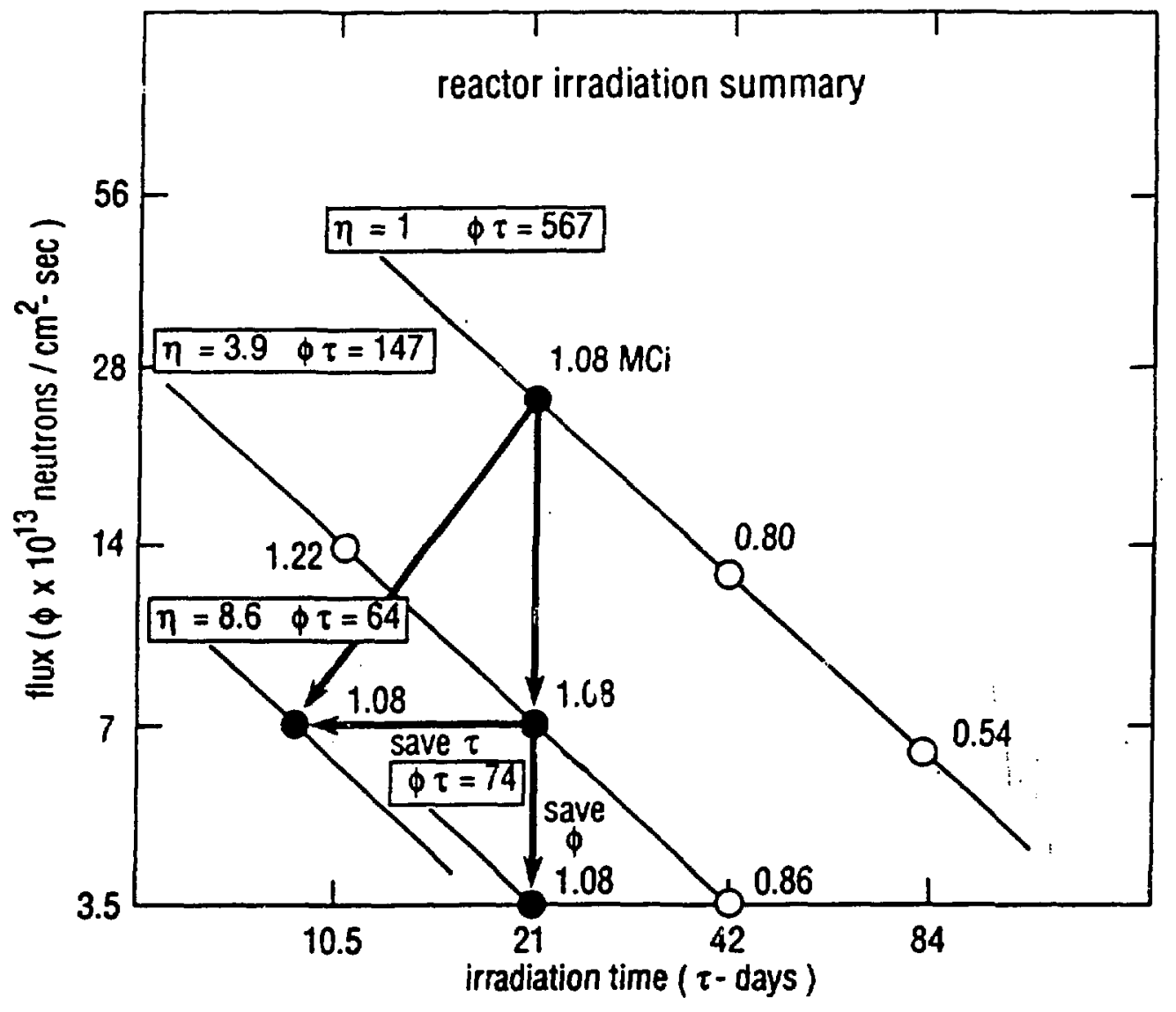

FIG. 3 Reactor irradiation summary. The activity of various neutrino sources is plotted as a function of flux $\phi$ and irradiation time $\tau$. Lines of constant $\phi \tau$ are also depicted for three enrichment values $\eta$. The bold arrows connect sources all at the nominal level of $1.08 \mathrm{MCi}$. Enrichments factors, $\eta$, from 3.9 to 8.6 provide sources which can be conveniently prepared at the anticipated reactor fluxes and available irradiation times. 
irradiations is reduced by a factor of two, and the reactor irradiation time is doubled, the resulting two sources are only $0.80 \mathrm{MCi}$ due to the decay of $\mathrm{Cr}-51$ during the reactor irradiation. This is seen by following the uppermost line of constant "flux $x$ time" (the product of flux as multiples of $10^{13}$ times the time in days) in Figure 3. The other lines of constant flux-time product describe the activities produced by 3.9 -fold (17\% Cr-50) and 8.6-fold (37\% Cr-50) enrichments. The factor of four enrichment then produces the desired $1.08 \mathrm{MCi}$ source with $1 / 4$ the total reactor irradiation flux; this is the flux found when using the Siloe reactor. Once again, the detailed work is under study by the Grenoble and Saclay groups in GALLEX.

The same $1.08 \mathrm{MCi}$ source described above (4-fold enrichment, 21 days) could be achieved in half the irradiation time at roughly double the enrichment. The choice between these two options involves the financial tradeoff between enrichment costs versus the costs and availability of the nuclear reactor flux: In light of recent successful enrichment tests reported by Oak Ridge National Laboratory (ORNL) and summarized below, it is clear, even in the simple picture described, that strong desires exist to have a source at least in the 4-fold to 9-fold enrichment range. Ultimately, the availability of the Siloe reactor could be the most important factor.

\section{Enriched Chromium Source Preparations and Test Irradiations}

\section{A. The basic scheme.}

An intense Cr-51 neutrino source produced by neutron irradiation of enriched $\mathrm{Cr}-50$ is an essential part of the GALLEX project. A previous report (Ref. 13) discusses the irradiation test of purchased, natural chromium metal particles. As noted there in the conclusions, and in the previous discussions in Section II.C, the use of enriched chromium in the neutrino source significantly 
improves the calibration aspects of the GALLEX detector and currently is the route of choice.

The procedure for the preparation of an enriched chromium source that is adopted here begins with chromium of natural isotopic abundance in the form of the gaseous compound, chromyl fluoride, $\mathrm{CrO}_{2} \mathrm{~F}_{2}$. The isotope of $\mathrm{Cr}-50$ is enriched relative to the heavier chromium isotopes by passing it through two stages of gas centrifugation at ine Oak Ridge National Laboratory. Because the enriched material is in a gaseous form, a chemical procedure is required to convert the chromyl uoride gas to a high purity solid which is suitable for irradiation in the nuclear reactor. We developed these chemical steps at Brookhaven National Laboratory: the enriched chromyl fluoride is reacted with water to produce chromic acid in the $\mathrm{Cr}(\mathrm{VI})$ oxidation state; this acid is subsequently reduced to the hydrous $\operatorname{Cr}$ (III) oxide; this oxide is then fired in a furnace to form the very stable chromium(III) oxide, $\mathrm{Cr}_{2} \mathrm{O}_{3}$. Specific chemical steps in the reactions were chosen so as to use readily available reagents which are obtainable in high purity; a key to this source design is the need to minimize the presence of impurities. Some common impurities, even at the ppm level, as detailed later in Section IV, would be severely limiting. Specifically, we report on a study of test $\mathrm{Cr}-51$ sources which were produced using these procedures. These sources were irradiated in a nuclear reactor at BNL.

A subsequent report ${ }^{24}$ describes work done to investigate the materials properties of the chromium oxide. Particular attention in this follow-on report is paid to the study of the chromium grains in an to attempt to chemically influence the grain size produced during the high temperature firing. In addition, a chromium-ceramic composite is under investigation by us as a source material. A very different procedure is being examined by other members of the GALLEX Collaboration, namely, electrolysis ${ }^{14}$ whereby chromic acid is reduced directly to 
chromium metal. Hence, this report restricts itself to the work completed in preparing an enriched chromium source in the form of chromium(III) oxide.

\section{B. The enrichment test.}

Chromyl fluoride is a red-violet solid that sublimes to a reddish-brown gas at atmospheric pressure. 25 Engelbrecht and Grosse 26 report that the vapor pressure $\mathrm{P}$ in $\mathrm{mm}$ as a function of temperature $\mathrm{T}$ in $\mathrm{deg} . \mathrm{K}$ is given by:

$$
\log _{10} \mathrm{P}(\mathrm{mm})=6.25189+959 \mathrm{~T}^{-1}-599333 \mathrm{~T}^{-2}
$$

for the solid, and:

$$
\log _{10} \mathrm{P}(\mathrm{mm})=8.80494-1785.5 \mathrm{~T}^{-1}
$$

for the liquid (both over a "limited range"). The solid reaches a vapor pressure of $760 \mathrm{~mm}$ at $29.6^{\circ} \mathrm{C}$ and it melts at $31.6^{\circ} \mathrm{C}$. The triple-point vapor-pressure is $885 \mathrm{~mm}$. The data of vapor pressure versus temperature depicted in Fig. 4 indicate that $\mathrm{CrO}_{2} \mathrm{~F}_{2}$ is a suitable candidate gas for the chromium enrichment by gas centrifugation.

There are a number of uncertainties relevant to the use of chromyl fluoride in the gas-centrifuge system. Because of its oxidixing and fluorinating properties, it is possible for the gas to attack many common materials. Additionally, since some of the centrifuges may have been used for the enrichment of uranium in the isotope U-235, the possibility exists that any remaining uranium oxide deposits could be fluorinated to form $\mathrm{UF}_{6}$ and possibly picked up in the effluent gas. In order to investigate such potentially serious problems, we arranged a collaborative effort with Oak Ridge staff (A. Szady, W. L. Roberts and C. Liles) to do enrichment tests of the chromyl fluoride at ORNL. The emphasis in these tests was on the identification of levels of impurities. 


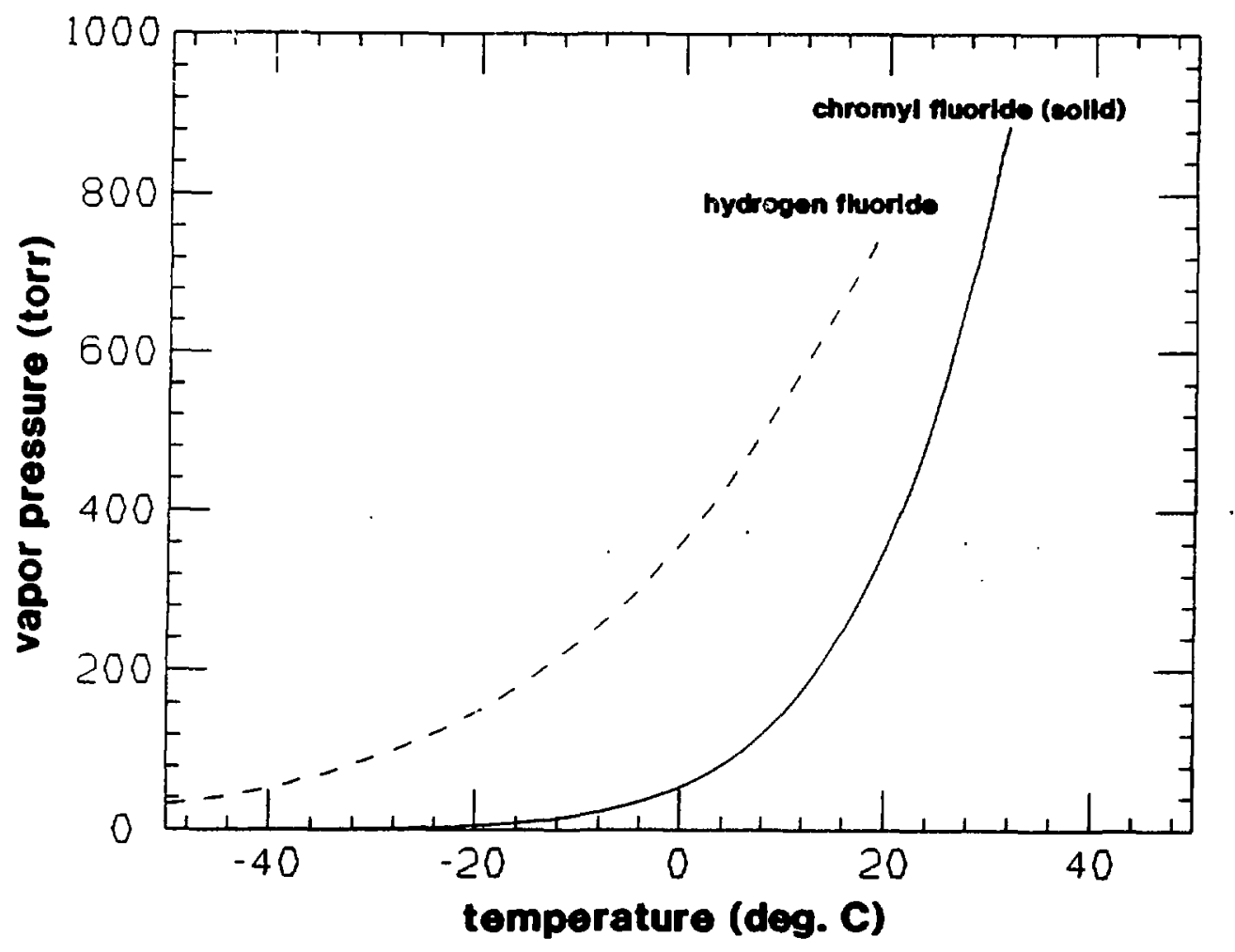

FIG. 4 Vapor pressures of $\mathrm{CrO}_{2} \mathrm{~F}_{2}$ and $\mathrm{HF}$. At room temperature, solid $\mathrm{CrO}_{2} \mathrm{~F}_{2}$ has a significant vapor pressure--at $29.6^{\circ} \mathrm{C}$, the vapor pressure is $760 \mathrm{~mm}$--thus making it a convenient gas for chromium enrichment. A bath of dry-ice acetone (around $-80^{\circ} \mathrm{C}$ ) is convenient to separate $\mathrm{HF}$ and $\mathrm{CrO}_{2} \mathrm{~F}_{2}$ fractions in $\mathrm{HF}$-contaminated gas. 
The enriching capabilities 27 of the ORNL centrifuges are summarized in Fig. 5. As illustrated there, two centrifuge passes were required in the test runs to reach the desired range of enrichment of $\mathrm{Cr}-50$. Note that these tests provided the first samples of chromium that were ever isotopically enriched by gas centrifugation.

Approximately 2000 grams of chromyl fluoride were purchased from the Ozark-Mahoning Company for use in the initial studies. From these tests three chromyl fluoride samples were produced at ORNL for conversion to chromium oxide at BNL. 'These are:

1. The enriched sample. This sample was produced by passing the "heads" (light fraction) from a first pass through the centrifuge back through the centrifuge a second time. The isotopic ratios were reported as:

Cr-50 --21.4\%, Cr-52 - 74.4\%, Cr-53 -- 3.9\% and Cr-54 - $0.5 \%$.

Samples prepared for neutron activation analysis using this material are labelled by the prefix number 1 (e.g. in Appendix A).

2. The leached sample. This sample was obtained by circulating chromyl fluoride through the centrifuges in order to intentionally pick up impurities. No isotope enrichment was performed. It is a mixed sample containing material from a number of tests at ORNL. Samples using this material are labelled by the prefix number 2 .

3. The feed gas. This sample is a batch of chromyl fluoride which failed to work in the centrifuges because it reportedly contained excessive amounts of hydrogen-fluoride gas. Limited studies were done on this material in a series of neutron-activation runs as described later. Samples using this material are labelled by the prefix number 3 . 


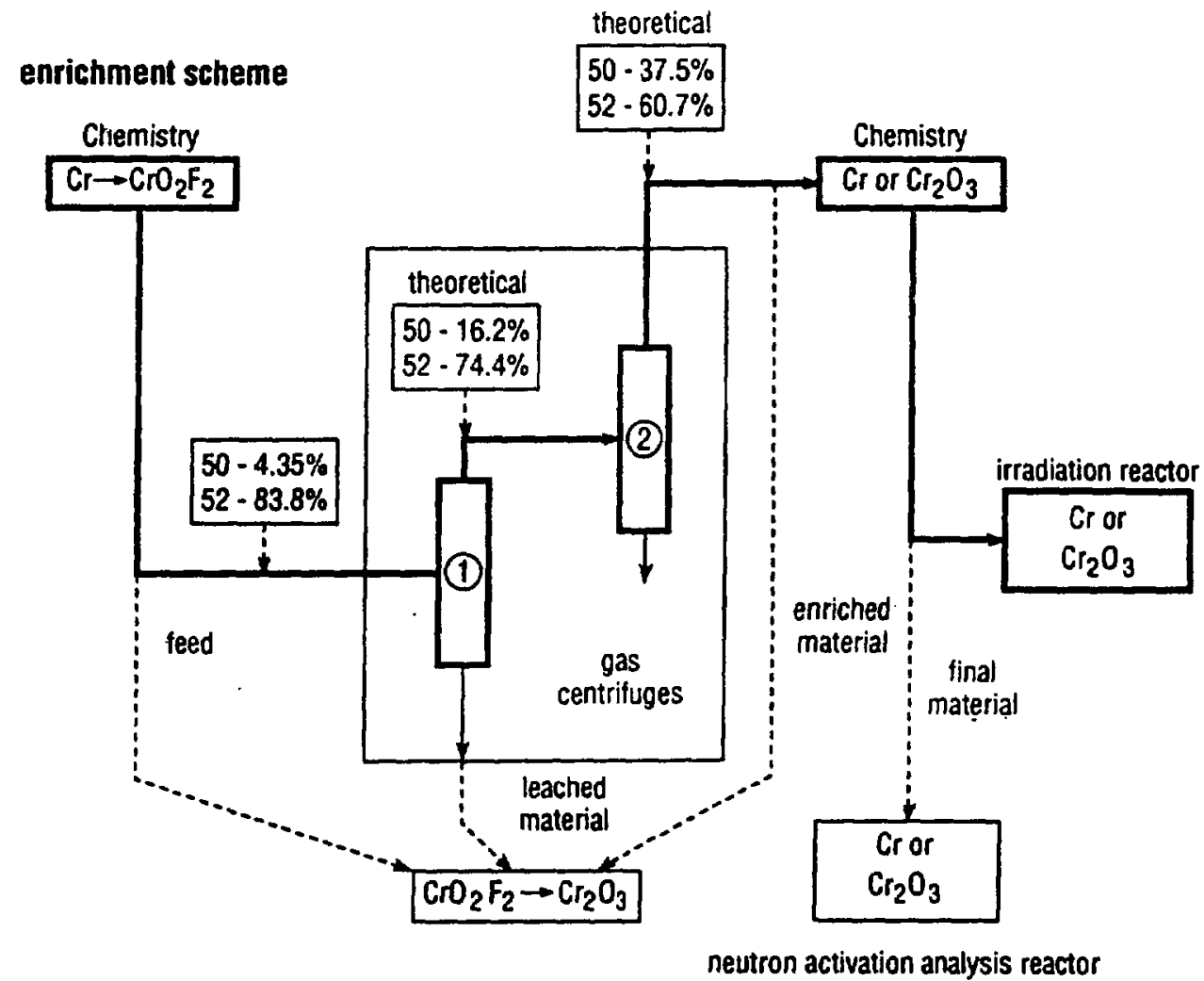

FIG. 5 Schematic of the enrichment test. Chromyl fluoride produced from natural chromium was enriched in $\mathrm{Cr}-50$ to $21.4 \%$ following two passes through the gas centrifuge. The theoretical values indicate the enrichment expected with optimization of the system's operating parameters (Ref. 27). Conversion to $\mathrm{Cr}_{2} \mathrm{O}_{3}$ is useful both in sampling throughout and testing the final source material. 
In addition to these three chroniyl fluoride samples based on the Ozark-Mahoning feed material, chromyl fluoride samples (a total of $10 \mathrm{~g}$ ) were also provided by the Advanced Research Chemicals company. Tests using samples of this material are labelled by the letters ARC.

\section{Conversion to enriched chromium oxide.}

The chromyl fluoride used in this work is produced commercially by the reaction of hydrogen fluoride (HF) with chromium(VI) trioxide $\left(\mathrm{CrO}_{3}\right)$. Preparation of chromyl fluoride in this manner is described by Engelbrecht and Grosse. 26 The relevant equilibrium is

$$
\mathrm{CrO}_{3}+2 \mathrm{HF} \longmapsto \mathrm{CrO}_{2} \mathrm{~F}_{2}+\mathrm{H}_{2} \mathrm{O}
$$

Upon addition of $\mathrm{CrO}_{2} \mathrm{~F}_{2}$ to excess water, the reverse reaction occurs. The resulting $\mathrm{CrO}_{3}$ when dissolved in $\mathrm{HF}$-free water obeys the following relevant reactions:

$$
\begin{array}{ll}
\mathrm{CrO}_{3}+\mathrm{H}_{2} \mathrm{O} \longmapsto \mathrm{H}_{2} \mathrm{CrO}_{4} \\
\mathrm{H}_{2} \mathrm{CrO}_{4} \longmapsto \mathrm{H}^{+}+\mathrm{HCrO}_{4}^{-} & \left.\mathrm{k}_{1}=\left[\mathrm{H}^{+}\right]\left[\mathrm{HCrO}_{4}^{-}\right] / \mathrm{H}_{2} \mathrm{CrO}_{4}\right] \\
\mathrm{HCrO}_{4}^{-} \longmapsto \mathrm{H}^{+}+\mathrm{CrO}_{4}^{-2} & \mathrm{k}_{2}=\left[\mathrm{H}^{+}\right]\left[\mathrm{CrO}_{4}^{-2}\right] /\left[\mathrm{HCrO}_{4}^{-}\right] \\
2 \mathrm{HCrO}_{4}^{-} \longmapsto \mathrm{H}_{2} \mathrm{O}+\mathrm{Cr}_{2} \mathrm{O}_{7}^{-2} & \left.\mathrm{k}_{3}=\left[\mathrm{Cr}_{2} \mathrm{O}_{7}^{-2}\right] / \mathrm{HCrO}_{4}^{-}\right]^{2}
\end{array}
$$

where:

$$
\begin{aligned}
& \mathrm{k}_{1}=4.1 \\
& \mathrm{k}_{2}=1.3 \times 10^{-6} \\
& \mathrm{k}_{3}=160
\end{aligned}
$$

The constants, $k_{1}, k_{2}$ and $k_{3}$ are obtained from Ref. 28 . In the presence of $H F$ the additional relevant equilibrium is

$$
\mathrm{k}_{4}=\left[\mathrm{H}^{+}\right]\left[\mathrm{F}-\mathrm{j} /[\mathrm{HF}] \quad \mathrm{k}_{4}=3.5 \times 10^{-4}\right.
$$


where $\mathrm{k}_{\mathbf{4}}$ is from Ref. 29. In the presence of fluoride ion (and chloride ion as noted below), the $\mathrm{HCrO}_{4}^{-}$is essentially quantitatively converted to $\mathrm{CrO}_{3} \mathrm{~F}^{-}$,

$$
\mathrm{HCrO}_{4}^{-}+\mathrm{H}^{+}+\mathrm{F}^{-} \rightarrow \mathrm{CrO}_{3} \mathrm{~F}^{-}+\mathrm{H}_{2} \mathrm{O}
$$

In the approach which we use, the chromyl fluoride is reacted with water. The resulting solution then contains varying amounts of $\mathrm{HF}$ acid. Next, the solution is evaporated to yield the solid product $\mathrm{CrO}_{3}$. This approach eliminates the presence of the fluoride ion which would form complexes with the $\mathrm{Cr}^{+3}$ species produced later in the reduction step. The remaining steps of the procedure are then based on the approach of Ref. 30. Excess $12 \mathrm{~N}$ aqueous $\mathrm{HCl}$ is added to the $\mathrm{CrO}_{3}$ solid to give the $\mathrm{CrO}_{3} \mathrm{Cl}^{-}$ion

$$
\mathrm{HCrO}_{4}^{-}+\mathrm{H}^{+}+\mathrm{Cl}^{-} \rightarrow \mathrm{CrO}_{3} \mathrm{Cl}^{-}+\mathrm{H}_{2} \mathrm{O}
$$

where the reaction is once again essentially quantitative.

The diagram in Fig. 6 serves to illustrate the regimes of total $\mathrm{Cr}^{+6}$ ion concentration and of $\mathrm{pH}$ where the various ionic species described by the above equilibria apply. An $\mathrm{HCl}$ concentration of $10 \mathrm{M}(\mathrm{pH}=-1)$ and ion concentration of $\left[\mathrm{Cr}^{+6}\right] \approx 0.1 \mathrm{M}$ mark the characteristics of the solution prior to the reduction step.

The highly acidic $\mathrm{Cr}^{+6}$ solution thus prepared is reacted with an ethanol-water solution. In this reaction the $\mathrm{Cr}^{+6}$ oxidizes the organic molecule and is reduced to $\mathrm{Cr}^{+3}$ according to the following illustrative stoichiometry,

$$
3 \text { ethanol }+12 \mathrm{H}^{+}+4 \mathrm{CrO}_{3} \mathrm{Cl}^{-} \rightarrow 4 \mathrm{Cr}^{+3}+4 \mathrm{Cl}^{-}+3 \text { "acetic acid" }+9 \mathrm{H}_{2} \mathrm{O}
$$

where "acetic acid" emphasizes the fact that an array of possible oxidation products exists. The $\mathrm{Cr}^{+3}$ is precipitated with a basic ammonia solution, to form the hydrous chromium oxide. This material is chemically written as $\mathrm{Cr}_{2} \mathrm{O}_{3} \bullet \mathrm{xH}_{2} \mathrm{O}$ or perhaps commonly as the chromium hydroxide " $\mathrm{Cr}(\mathrm{OH})_{3}$ ". We will keep the symbol $\mathrm{Cr}_{2} \mathrm{O}_{3} \bullet \mathrm{xH}_{2} \mathrm{O}$ to refer to the unfired precipitate. The final 


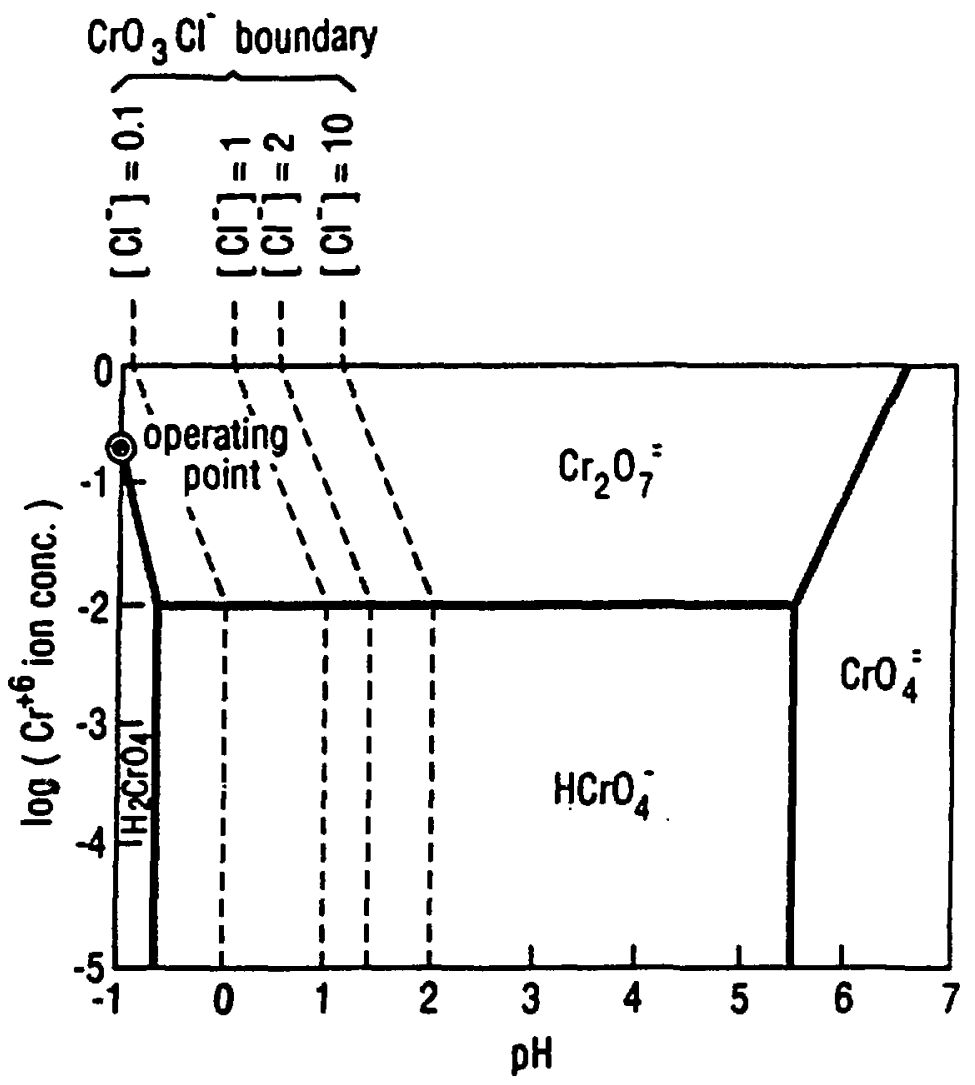

FIG. 6 Equilibria between ionic chromium species. The chromium (VI) ion is in the form $\mathrm{CrO}_{3} \mathrm{Cl}^{-}$following the elimination of $\mathrm{HF}$ and addition of $\mathrm{HCl}$. The "operating point" marks the conditions of $\mathrm{pH}$ and $\left[\mathrm{Cr}^{+6}\right]$ prior to the reduction to Cr (III). 
anhydrous $\mathrm{Cr}_{2} \mathrm{O}_{3}$ product is then produced by firing the chromium hydroxide in a furnace, typically above $800 \mathrm{deg}$. C. Studies described below in III.F involved some furnace firings which were carried out up to $1200 \mathrm{deg}$. $\mathrm{C}$, the highest temperature conveniently available in the typical furnaces.

The specific steps and experimental approaches are detailed in the remaining parts of this section. These describe the piping system used to handle the chromyl flaoride, the wet chemistry and high purity reagents, and finally the firing procedures.

\section{Apparatus to handle chromyl fluoride.}

An apparatus was designed and built to allow sampling of chromyl fluoride from shipping bottles, to facilitate its transfer by cryogenic pumping and to carry out its reaction with water. The apparatus was designed so as to maximize the use of quick-disconnect couplings to permit cleaning and easy changing of piping configurations. A schematic of the apparatus appears in Fig. 7.

The apparatus is built out of monel, stainless steel, Kel-F, Teflon and copper parts. Monel is an alloy of nickel and copper that is most resistant to attack by fluorine compounds; consequently, the main header and its associated valves are made of monel. The appendages to the main header that are used to store, react, distill and handle the chromyl fluoride are fabricated from stainless-steel off-the-shell parts for expediency. Monel would ultimately be a better choice for long term durability, but it was found that the chromyl fluoride attacked only wetted stainless-steel surfaces. The remainder of the system's piping which is used to provide for traps, nitrogen pressurization and vacuum services was constructed primarily of stainless steel with some pieces made of monel. 


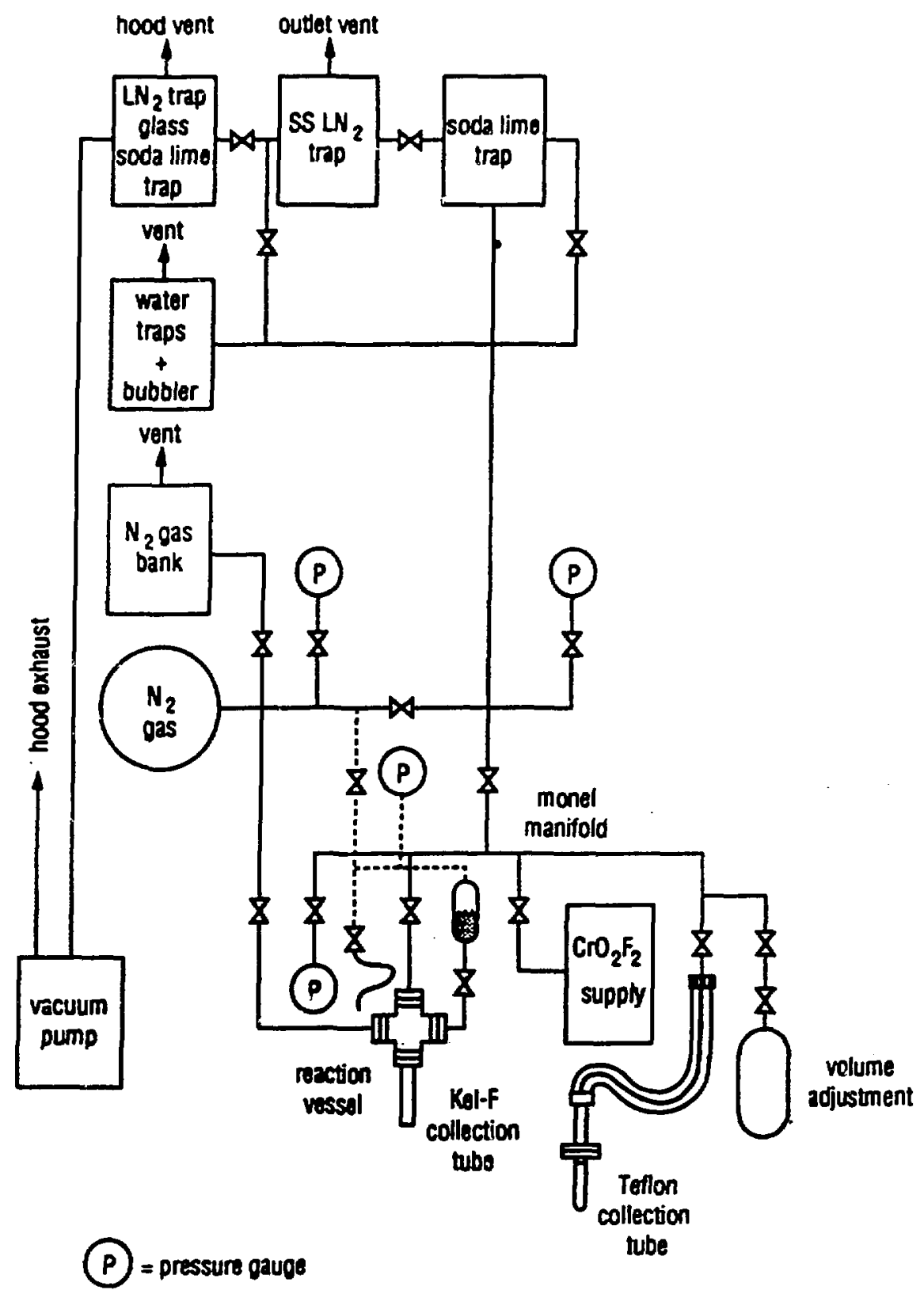

FIG. 7 Schematic of the chromyl fluoride sampling apparatus. The apparatus is used to sample chromyl fluoride gas and convert it to $\mathrm{Cr}_{2} \mathrm{O}_{3}$ for reactor irradiations. 
Various lines which vent sections of the apparatus are made of copper tubing, as these lines may be exposed to gases such as HF during routine use. The nitrogen pressurizing section, depicted in the lower left corner of Fig. 7, is made of brass as this piping is intended to be exposed to vacuum or nitrogen gas only.

Two types of traps are employed to prevent the chromyl fluoride from entering the vacuum pump or the atmosphere; liquid-nitrogen-filled traps and soda-lime traps. Both of these traps are duplicated for redundancy; the main traps are made of stainless steel and the backup traps are made of glass. The glass trap filled with soda lime, in particular, provides an opportunity for one to visibly inspect for any chromyl fluoride after the two main traps and prior to the final liquid-nitrogen trap, also made of glass.

The larger, main, soda-lime trap contains a perforated metal plate internally positioned near the inlet at the base of the trap that prevents the soda lime from fallirg into the inlet piping. This piping is also stuffed with glass wool. A long connecting rod is screwed into the plate and can be used to pull out the soda lime for replacement; the internal assembly thus acts as a piston.

The stainless-steel liquid-nitrogen trap downstream of the soda-lime trap is intended to be a backup to the soda-lime trap and to improve the vacuum in the metal section. Both of these traps include connections to a copper venting-line. In particular, if it is necessary to vent the stainless-steel liquid-nitrogen trap by allowing it to warm up, this can be achieved through the vent line. In some reactions where it is desirable to keep the reaction vessel near atmospheric pressure under a nitrogen flow, the effluent nitrogen, which potentially contains $\mathrm{HF}$, passes through the venting line and into a bubbler after exiting the main soda-lime trap. The bubbler is filled with water and an acid-base indicator (phenolphthalein) to detect any leakage of chromyl fluoride. 
The nitrogen-pressurizing section contains both a bypass for rapid filling of the metal system, and an adjustable flow meter. Five storage banks, each about one liter in volume, are filled with nitrogen to provide a buffer to the atmosphere when bringing the system up to atmospheric pressure with nitrogen gas. This feature is a safety feature to insure that the reaction vessel is not over-pressurized when establishing a nitrogen flow through it. The bubbler referred to above also provides a flow indicator as a back-up to the indication given by the inlet flow meter.

A cross-connect valve connects the metal system directly to the nitrogen section. Although this connection is used for rapid filling of the metal section, it is also used to draw a vacuum in the nitrogen section prior to filling with nitrogen. Nitrogen is also supplied to one of the inlet tubes of the reaction vessel and to the pressurized addition chamber that is used to force water into the reaction vessel.

The reaction vessel consists of a stainless-steel flange with three welded tubes. One valved tube connects the vessel to the metal section to receive chromyl fluoride. The second valved tube serves as a nitrogen inlet, which is used when a flow is established through the vessel. In this instance the outlet is through the same connection to the metal system that is used to sample chromyl fluoride. A third valved tube connects the vessel to the addition chamber, to be described below. The reaction-vessel seal consists of two knife-edge flanges which grip a removable Kel-F or Teflon insert. The lip of the plastic insert, which serves as the seal, is somewhat thick (approximately $5 \mathrm{~mm}$ ) to allow refurbishing if necessary. A similar arrangement is used in the distillation vessel, to be described in section III.E.

In some sampling runs, a nitrogen-pressurized chamber was used to add water to a solidified sample of chromyl fluoride. This addition chamber consists 
of a cylindrical metal tube. The top of the tube has two valved connections. The upper valve is connected to the nitrogen section, so the addition chamber can be evacuatea through the nitrogen section to the vacuum section. A lower valve is connected to a teflon tube; it is then used to draw in a measured amount of water from a graduated cylinder. The nitrogen section is then pressurized such that the upper valve is now used to pressurize the chamber, providing the force needed to push the water into the reaction vessel.

\section{E. Sampling procedure.}

The basic sequence used in producing chromium oxide samples is to draw a sample at room temperature from a chromyl fluoride bottle into the evacuated, but isolated, metal header by opening the valve to the sample bottle and observing the pressure increase from vacuum to approximately $11.6 \mathrm{in} . \mathrm{Hg}$ (the gauge units) or 466 torr. A $150-\mathrm{ml}$ empty stainless-steel bulb is attached to the header so as to adjust the total gas volume at this point in the procedure to $243 \mathrm{ml}$. This volume typically corresponds to 6.1 millimoles of $\mathrm{CrO}_{2} \mathrm{~F}_{2}$ or $320 \mathrm{mg}$ of $\mathrm{Cr}$ at room temperature. This will subsequently produce, at $100 \%$ yield, $610 \mathrm{mg} \mathrm{CrO}_{3}$ or $460 \mathrm{mg}$ of $\mathrm{Cr}_{2} \mathrm{O}_{3}$, ideal amounts for use in the reactor irradiations.

The chromyl fluoride gas, thus sampled, is then cryogenically collected completely in the distillation vessel using liquid nitrogen. This vessel consists of a Kel-F tube with flanged connection, as described in the previous section. The Kel-F tube is translucent and one can see the red chromyl fluoride as it collects. Next, the unneeded sections of the main monel header are isolated and the distillation vessel is evacuated for 15 minutes after immersing it in a dry-ice

acetone bath. At this bath temperature $\left(-78^{\circ} \mathrm{C}\right)$, the vapor pressure of the $\mathrm{HF}$ is appreciably higher than that of the chromyl fluoride (see Fig. 4) and any notable amounts of $\mathrm{HF}$ can be removed at this stage. 
The chromyl flouride material thus collected is then cryogenically pumped into the reaction vessel, once again using liquid nitrogen as coolant. As discussed later, this approach was found to introduce unwanted impurities from the stainless-steel walls of the addition tube, primarily due to the adherence of the chromyl fluoride to the walls; upon the addition of water to the sample, the fluoride attacks the stainless steel. To solve this problem, part of the stainless-steel tube could be replaced by an appropriately resistant plastic. Instead, we tried an alternate procedure whereby a nitrogen flow carries the chromyl-fluoride gas into a vessel of water through a section of teflon tubing. This approach also did not work well since the chromyl fluoride tended to clog the tip of tube and some gas would bubble through the solution without reacting.

The approach that we finally used is one in which the chromyl fluoride is cryogenically pumped through a lengthy section of teflon tubing into a teflon vessel. This vessel is capped by a teflon insert, with a hole through which the teflon tube can be inserted so as to project approximately $5 \mathrm{~mm}$ into the teflon vessel. This tube in turn is wholly contained within a stainless-steel flexible tube connected to the monel header at one end and to a knife-edge flange at the other end. The teflon tube then inserts into the capped teflon vessel which is in turn forced up against the knife-edge seal, producing a vacuum tight unit. A totally encased teflon assembly thus results. The chromyl fluoride is then transferred into this vessel by cryogenic pumping. In the current configuration, while the teflon vessel is still cold, the knife-edge seal is loosened, the teflon tube is unplugged, and water is added to the solid through the hole in the teflon cap. This approach was found to work successfully for these small samples.

Stoichiometrically, only $0.1 \mathrm{ml}$ of water is necessary for the complete reaction of the chromyl fluoride. However, we use a large excess of water, $5 \mathrm{ml}$, to insure a rapid reaction with all of the material. In situations where we produce a 
blank to check the impurities of the reagents, $610 \mathrm{mg}$ of reagent-grade $\mathrm{CrO}_{3}$, instead of the $\mathrm{CrO}_{2} \mathrm{~F}_{2}$, are added to $2.0 \mathrm{ml} \mathrm{H} \mathrm{H}_{2} \mathrm{O}$. To this chromic acid solution, we then add $3.0 \mathrm{ml}$ of a $4.1 \mathrm{M} \mathrm{HF}$ solution ( $14 \mathrm{ml}$ concentrated HF solution dissolved in $100 \mathrm{ml} \mathrm{H} \mathrm{H}_{2} \mathrm{O}$ ). This blank sample is then carried through the remaining steps of the procedure as if it had been produced from chromyl fluoride.

The concentrated solution essentially contains the $\mathrm{CrO}_{3} \mathrm{~F}^{-}$ion and $\mathrm{HF}$ at this point in the chemical procedure. Next, the solution is evaporated to dryness by fitting the teflon vessel into a quartz holder and heating the holder under a heat lamp. The teflon insert used when filling the tube with chromyl fluoride is removed and replaced by a fitted perforated teflon cap to allow the evaporation to proceed. A teflon beaker is positioned over the quartz assembly such that the condensed water vapor and dissolved HF collects inside the beaker surface and drips into a teflon tray. The crystalline $\mathrm{CrO}_{3}$ is now ready for the reduction step.

\section{F. Reduction and firing of $\mathrm{Cr}_{2} \mathrm{O}_{3} \cdot \mathrm{XH}_{2} \mathrm{O}$ to produce high purity $\mathrm{Cr}_{2} \mathrm{O}_{3}$ -}

Two characteristics of the chemical reagants used in these procedures are very important: (1) they must not contain (metallic) impurities that would give rise to unacceptably high levels of radioactivity when the source is irradiated, and (2) they should be inexpensive since large amounts are used. For these reasons, it was decided to use hydrochloric acid and ethyl alcohol for the reduction step and ammonia for the precipitation steps, as outlined in the previous section, III.E. There is no perceived problem in replacing these reagents with other reagants equally pure, especially the alcohol.

The elements in the typical reactants sturied here are oxygen, hydrogen, chlorine, carbon and fluorine. Carbon, containing $1.11 \% \mathrm{C}-13$, produces $5730 \mathrm{y}$ $\mathrm{C}-14$; and chlorine, containing $75.77 \% \mathrm{Cl}-35$ produces $3.00 \times 10^{5}$ y $\mathrm{Cl}-36$. The remaining elements produce very short-lived activities. Moreover, by utilizing 
water, ammonia, hydrochloric acid and ethanol, one uses reagents all of which are obtainable in highly pure forms by distillation. Consequently it is simple to design a clean radiochemical procedure.

To do the reduction, the $\mathrm{CrO}_{3}$ solid is added to about $10 \mathrm{ml}$ of high purity water, triply distilled, in a bakeable teflon beaker. The beaker has a graphite impregnated base which can be heated on a hot plate. The teflon beaker is used to lessen the likelihood of picking up any impurities during the course of the reaction since the initial solution is highly corrosive. To this solution is added $10 \mathrm{ml}$ of concentrated $\mathrm{HCl}$, then $70 \mathrm{ml}$ of ethanol. The reaction proceeds very slowly at room temperature, turning from the bright orange color of the chromate ion to a dark brownish color. The beaker is then placed on a stirring hot plate and heated to a boil for approximately 15 minutes. At this point the solution is the grassy green color of the $\mathrm{Cr}^{+3}$ ion. A concentrated ammonia solution is added dropwise until the blue-gray hydrous chromium oxide forms in the beaker. The solution is brought to a $\mathrm{pH}$ of 8 . It is not desirable to make the solution more basic because the hydrous chromium oxide will redissolve, indicated by a violet color. Approximately $30 \mathrm{ml}$ of water are added to the beaker and heating to near boiling is continued for an additional 10 minutes to help coagulate the material.

The resulting hydrous chromium-oxide precipitate is very fine. In order to aid in the collection of the precipitate, the chromium-oxide suspension is then added to a large size (approx. $1000 \mathrm{ml}$ ) beaker containing $\sim 700 \mathrm{ml}$ of water and allowed to stand overnight. During this time the precipitate settles to the bottom, after which the supernatant is siphoned off. The procedure is repeated twice.

The solid is left in the same glass beaker, covered with a watch glass and placed in a drying oven at $110^{\circ} \mathrm{C}$ for typically two days. The partially dried material is next transferred to covered aluminum-oxide crucibles for firing in a 
furnace. The firing procedure drives off the bound water and ammonium chloride and allows for the growth of the chromium-oxide crystals.

In the furnace, the hydrous chromium oxide is heated to temperatures up to $1200 \mathrm{deg}$. $\mathrm{C}$ for typically 15 hours. Since the temperature and the atmospheric conditions in the furnace can affect the crystal-grain size, studies were carried out to investigate these effects. These studies are described in a subsequent report dealing with materials properties. ${ }^{24}$ The present section describes the treatment of the samples used for the reactor irradiations.

Samples used for neutron-activation analyses are fired in a programmable Lindberg oven. The floor of the oven is covered with aluminum-oxide powder which protects the oven floor in the event of a release of material from the crucible. The samples are heated to 200 degrees for 30 minutes to drive off any gross amounts of water, then to 300 degrees for 30 minutes to continue the drying and finally to $\mathbf{3 5 0}$ degrees to 400 degrees to drive off residual ammonium chloride. The temperature is stepped up in this manner to avoid the rapid production of gasses which would cause the material to erupt from the crucibles. After approximately 30 minutes at 350 to 400 degrees, the oven is heated to 800 degrees or more, a process which takes about two hours. The samples are then heated at the maximum temperature for approximately 15 hours. After cooling in a dessicator, the chromium oxide appears as a dark green powder. During subsequent heating, done for other work, it is possible to observe the discoloration of the green powder to a slight brownish due to the production of some $\mathrm{Cr}^{+6}$ at the elevated temperatures.

In a typical sample, about $2.01 \mathrm{mmol}$ or $201 \mathrm{mg}$ of $\mathrm{CrO}_{3}$ is produced from the chromyl fluoride. This amount gives $350 \mathrm{mg}$ of the hydrous chromium oxide contaminated with some ammonium chloride. After firing, $1.68 \mathrm{mmol}$ of $\mathrm{Cr}$ in the form of $128 \mathrm{mg}$ of $\mathrm{Cr}_{2} \mathrm{O}_{3}$ are produced. This overall yield of $84 \%$ typifies the 
sample procedure used here and, although the materials were handled so as to minimize losses, no further work was done to improve the yields.

\section{G. Summary of reactor irradiations}

A sequence of four irradiations was carried out at the Brookhaven Medical Research Reactor: a summary of the irradiated samples appears in Appendix A.

1. 23 August 1989: Investigation of impurity levels of reagent-grade chromium oxide, $\mathrm{Cr}_{2} \mathrm{O}_{3}$, to establish handling techniques and set up the gamma-ray data processing. The irradiation lasted 1 hour at the BMRR at a flux level of $1 \times 10^{13}$ neutrons $/ \mathrm{cm}^{2}$-sec (a power level of $3 \mathrm{MW}$ ).

2. 31 October 1989: Investigation of impurity levels of chromium oxide samples produced from the ORNL-supplied chromyl fluoride. The irradiation lasted 6 hours at $3 \mathrm{MW}$.

3. 10 January 1990: Investigation of a procedure revised to minimize corrosion of the system used to prepare chromium oxide from the chromyl fluoride. The run lasted 3 hours at a variable flux. This run was in concert with other experiments at the BMRR and, for purposes of the test, the exact power levels were unimportant.

4. 12 February 1990: Investigation of impurity levels of chromium oxide prepared from both the ORNL samples and from the Advanced Research Chemicals (ARC) company.

\section{H. Samples and counting.}

Prior to the irradiations, the samples are loaded into ultra-high-purity quartz tubes ("supersil"), which are then sealed by the glass blower. The samples are then collected into groups of 4 to 6 so chosen that the groups each contain an assortment of samples and a blank or calibration standard. Each such group is 
wrapped in tissue paper with differing numbers of thin bands of aluminum foil to assist in identification of the bundles.

After irradiation, the surface of each quartz ampoule is cleaned by sequential immersion in concentrated HF, concentrated nitric acid and in triply distilled water. Each quartz ampoule is then loaded inside a glass vial. Empty glass vials were intentionally dropped to see how easily they broke. The drop tests indicated that they were strong enough for routine laboratory use.

Due to the high intensity (typically a few hundred counts/min-mg) of the $320-\mathrm{KeV}$ gamma ray emitted by $\mathrm{Cr}-51$, it was necessary to utilize lead shielding to diminish the intensity of this radiation so that gamma-rays from impurities could be detected. Taking into account the counting capabilities of the detector, the size of the samples, the duration of the reactor irradiations and our strong desire to observe the shorter-lived isotopes that would be important after three days of counting, we found that lead thicknesses in the range 0.3 to $1.0 \mathrm{~cm}$ were optimal at the given position in the gamma-ray detector cavity. The sample vial sits inside a lead house which can be reproducibly positioned at fixed distances from the detector head. The house significantly limits the exposure to the experimenter. A hole is drilled through one side of the house so as to fully expose the source to the detector while at the same time minimizing the scattered radiation that reaches the detector. The lead absorbers are then placed in front of this hole.

An intrinsic-germanium-crystal detector is used to obtain the gamma-ray spectra in the region of 0 to $3 \mathrm{MeV}$. The spectral data are recorded using a Nuclear Data ND66 multichannel analyzer and are transferred to a VAX computer. The spectra are analyzed by the computer code INTRAL (Ref. 31) to produce a "peaks file". A second computer program then produces the impurity levels and their uncertainties for each detected gamma ray. That is, for each gamma ray, a listing of the possible major isotopes is given along with the 
corresponding impurity levels of the natural elements. Simple analysis of this output is used to determine the impurity levels of the samples. Some isotopes, for example U-235, require special analyses, as described below. The efficiency correction for the location of the sample inside the lead-house and a check of the geometry of the transmission through the lead-house opening were carefully made as well. The reactor flux was checked using irradiation standards. Experimental checks indicated that the attenuation of observed gamma rays in the ampoule and glass vial were negligible, as was the attenuation by a thin aluminum absorber placed over the lead-house viewing port.

The impurity limits reported here take into account all aspects of the counting, including background levels where appropriate, and decay of the isotope during the counting period. A good discussion of neutron activation analysis is contained in Ref. 32 .

In Fig. 8 we show the spectrum of perhaps key interest. This figure depicts the gamma-ray spectrum of enriched Cr-50 (samples 6,7,8--Irradiation 4 in Appendix A) following the fourth irradiation, 6 hours at a flux of $1 \times 10^{13}$ neutrons $/ \mathrm{cm}^{2}$-sec, recorded 10 days after the reactor irradiation for a 32 minute counting time. Although quantitative information on the characteristics of the detector efficiency and lead absorbers is known and used in reporting impurity limits, as listed below, qualitatively we can say that the attenuation characteristics of the detector and the absorber for the selected geometry roughly cancel such that the peak intensities are roughly independent of gamma-ray energy. The major detected activity evident in this particular spectrum is due to lanthanum. The count rate of the chromium $320-\mathrm{keV}$ peak is 263 counts/min-mg absolute, whereas there are 0.0030 counts/min-mg in the lanthanum peak at $1596 \mathrm{MeV}$. Thus a clean source is obtained that is certainly suitable for making 


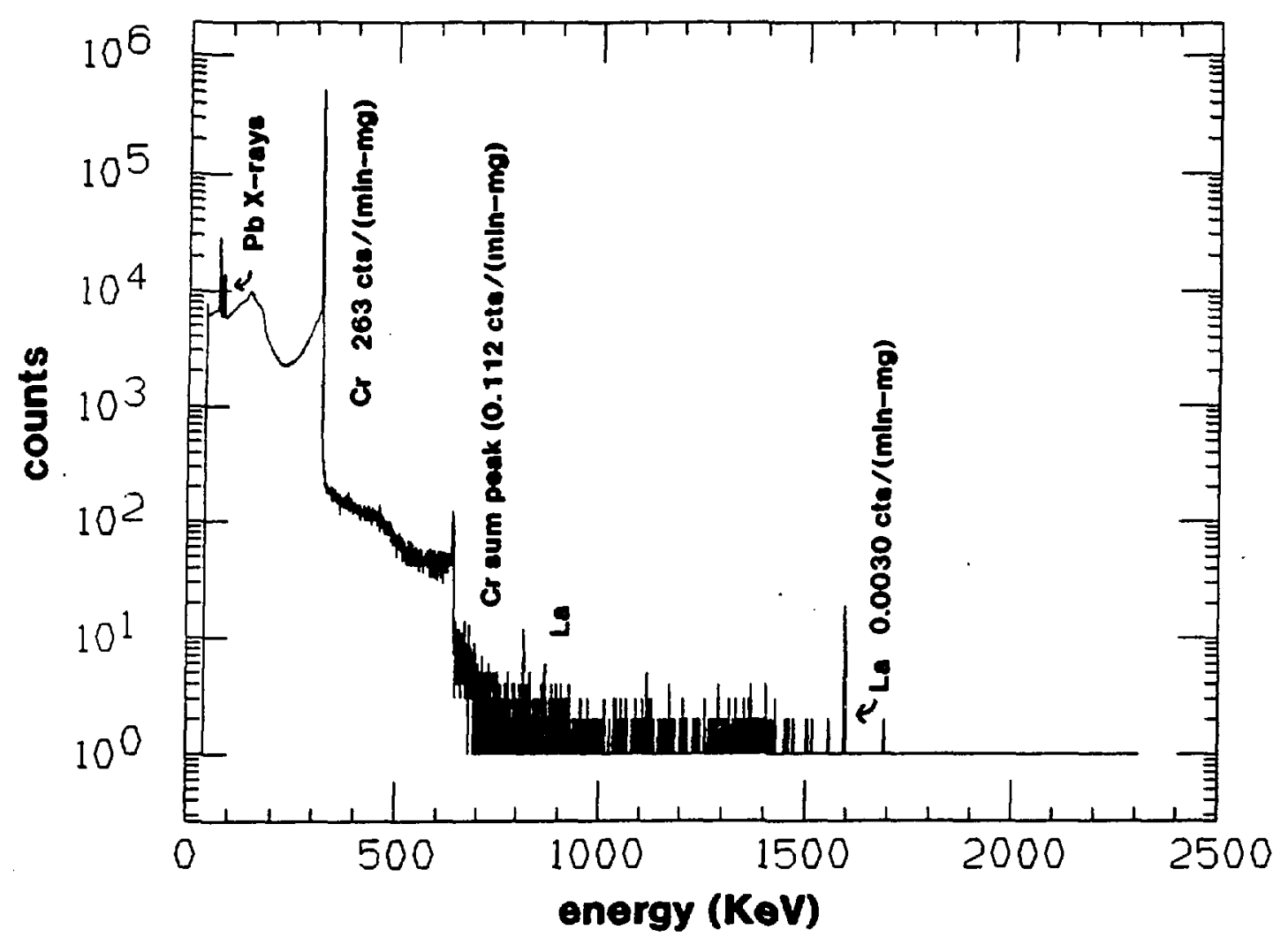

FIG. 8 Gamma-ray spectrum of an enriched Cr-51 source counted for 32 minutes on the tenth day after an irradition. 
the enriched-chromium neutrino-source. The complete details are continued below, but it is safe to say that no significantly limiting impurities are thus far seen.

\section{Enrichment results}

From the analysis of the count rates of Cr-51 activities averaged over the 3 samples we report the enrichment factors indicated in the Table 1 below.

\begin{tabular}{ll} 
Table I. \\
\multicolumn{2}{l}{ Chromium-50 Enrichment Results } \\
\hline Cr Sample & enrichment factor $(\eta)$ \\
\hline enriched & $5.18 \pm 0.38$ \\
leached & $0.84 \pm 0.05$ \\
ARC & $1.03 \pm 0.04$ \\
$\mathrm{CrO}_{3}$ & $0.993 \pm 0.061$ \\
$\mathrm{Cr}_{2} \mathrm{O}_{3}$ & $0.985 \pm 0.056$ \\
\hline
\end{tabular}

It is useful to note that the percentage of $\mathrm{Cr}-50$ in the enriched sample, $22.5 \%$, is in good agreement with the mass spectroscopy result, $21.4 \%$, reported by ORNL. The errors listed above are primarily due to counting statistics. Counting times were limited more by the number of samples and extremes of activity levels in each sample than by the desire to achieve longer counting times on the chromium emission, which was in most cases excessively attenuated by the lead shielding.

The listing for $\mathrm{CrO}_{3}$ refers to $\mathrm{Cr}_{2} \mathrm{O}_{3}$ sources made from reagent-grade $\mathrm{CrO}_{3}$ of natural isotopic abundances and thus serves as a chemical blank. The last listing refers to reagent-grade $\mathrm{Cr}_{2} \mathrm{O}_{3}$ samples. From these data it can be seen that 
the $\mathrm{Cr}-50$ in the leached material is clearly below the level found in natural chromium materials. This is certainly possible since the chromyl fluoride used in this sample was a mixture of various sources that went through the gas centrifuges. The numbers reported are absolute in the sense that the computer code includes a calibration number for the Cr-51 $320-\mathrm{KeV}$ line; thus the fourth and fifth chromium samples listed in Table 1 are in agreement with the chromium standards used in the development of the analyses code.

\section{J. Impurity analysis results}

The major concern in the Cr-51 neutrino-source design is the level of the impurities that result in long-lived activities which, in turn, emit energetic gamma rays. In their paper, Cribier et. al. 13 report on the long-lived activities produced in chromium grains which had been purchased from the manufacturer. We have specifically sought to measure these same impurities as well as any other possible long-lived activities $\left(t_{1 / 2}>1\right.$ week). It is, though, important for us not to ignore short-lived activities $\left(t_{1 / 2}<1\right.$ week) since these can arise from very common ions, such as sodium, which despite their shorter half-lives, produce notable activity levels during the first week following the irradiation. Still, it is the long-lived activities which are over-ruling and these are discussed first; uranium which potentially may be picked up from the walls of the gas centrifuges, is then considered; and finally, the short-lived activities are discussed. A convenient listing of the isotopes and relevant nuclear data, which we studied are summarized in Appendix B.

The significance of these results to the production of an intense $\mathrm{Cr}-51$ neutrino-source will be discussed below in Section IV. 


\section{Long-lived activities.}

The samples were studied to determine the impurities due to long-lived activities. A sample spectrum of the long-lived impurities in the enriched sample, measured ten days after the irradiation for a counting period of 3 days, is depicted in the figure below, Fig. 9. This figure represents a longer duration count of the same sample depicted in Fig. 8. The impurity levels detected, in parts per billion, are summarized in Table II for all of the $\mathrm{Cr}$ samples. The impurities, in units of $\mathrm{ppb}$, are listed in rough order of the potential severity of the impact of that isotope if it were in the final source. Thus scandium is one of the least desirable impurities, whereas iron is much more tolerable.

Table II. Summary of Detected Long-Lived Impurities in $\mathrm{Cr}_{2} \mathrm{O}_{3}$ Samples Produced from $\mathrm{CrO}_{2} \mathrm{~F}_{2}$ (in parts per billion).

\begin{tabular}{llcccc}
\hline Element & $\begin{array}{c}\text { Nuclide/ } \\
\text { Half-life }\end{array}$ & $\begin{array}{c}\text { Enriched } \\
\text { Samples }\end{array}$ & $\begin{array}{c}\text { Leached } \\
\text { Samples }\end{array}$ & $\begin{array}{c}\text { ARC } \\
\text { Samples }\end{array}$ & $\begin{array}{c}\text { Chemical } \\
\text { Blank }\end{array}$ \\
\hline scandium & Sc-46/84d & $4.9 \pm 3.1$ & $14 \pm 0.3$ & $6.4 \pm 0.2$ & $20 \pm 0.3$ \\
cobalt & Co-60/1900d & $7.6 \pm 6.3$ & $65 \pm 4$ & $37 \pm 3$ & $54 \pm 4$ \\
antimony & Sb-124/60d & $49 \pm 18$ & $200 \pm 30$ & $91 \pm 4$ & $200 \pm 10$ \\
tantalum & Ta-182/115d & $16 \pm 4$ & $15 \pm 2$ & $8.5 \pm 1.7$ & $7.5 \pm 1.8$ \\
uranium & U-235 & $2000 \pm 40$ & $160 \pm 20$ & $490 \pm 30$ & $890 \pm 50$ \\
& fission & & & & \\
& products/ & & & & \\
variable & & & & & \\
zinc & Zn-65/244d & $8800 \pm 200$ & $3100 \pm 100$ & $2700 \pm 100$ & $3400 \pm 100$ \\
iron & Fe-59/45d & $49000 \pm 3000$ & $25000 \pm 2000$ & $28000 \pm 2000$ & $31000 \pm 2000$ \\
\hline
\end{tabular}

\section{Results on uranium levels.}

A concern exists that uranium present in centrifuges used to enrich uranium hexafluoride in the useful U-235 isotope could make its way into 


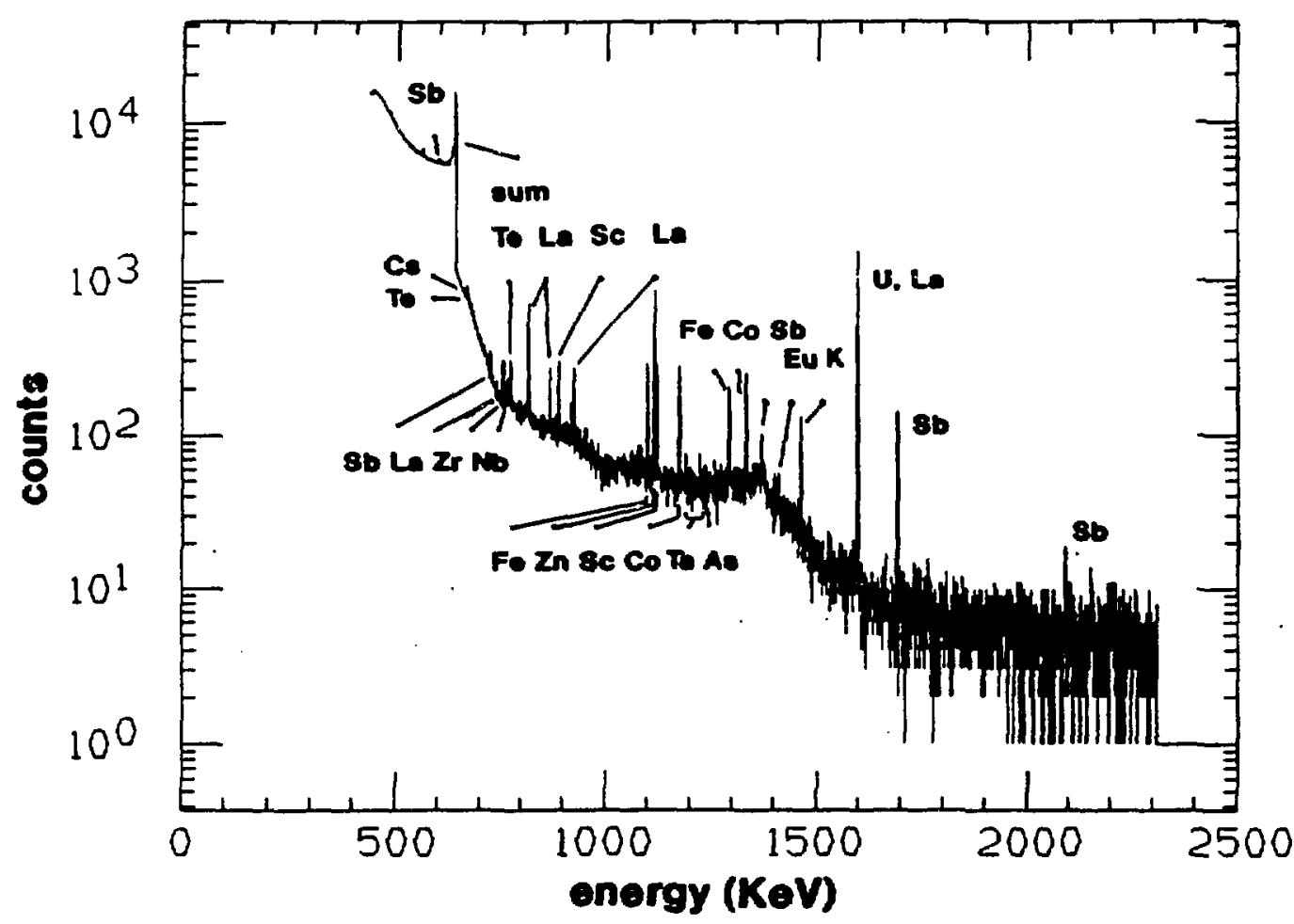

FIG. 9 Gamma-ra: spectrum of an enriched $\mathrm{Cr}-51$ source indicating long-lived $\left(t_{1 / 2}>1\right.$ week $)$ activities as measured starting 10 days after the irradiation. A number of peaks not annotated as well as $\mathrm{Zr}$ and $\mathrm{Nb}$ are attributed to U-235 thermal-neutron fission. 
enriched chromyl-fluoride gas through fluorination of any uranium deposits. Clearly the use of an enrichment process which has seen no exposure to uranium lessens the likelihood of this event. Ref. 33 reports on initial identification of uranium in chromyl fluoride samples.

In Fig. 10 we depict the activity from the enriched source, the uranium standard and the background quartz. The figure highlights the La peaks in the irradiated enriched sample due to the fission of U-235. The uranium impurity levels are listed in Table II.

The three approaches that were used to identify the uranium content are:

a) Comparison to an irradiated uranium standard.

b) Determination of Te-132 fission-product activity.

c) Determination of $\mathrm{Ba}-140$ fission-product activity.

The determination of the uranium by the third method, $c$, requires one to wait until any La-140 intially produced from La-139 decays such that only La-140 from Ba-140 decay is detected. All three methods proved very useful and were found to be in agreement. The uranium impurity limit is reported as "ppm natural uranium" (assuming $0.72 \%$ U-235 in natural uranium), in Table II to avoid any ambiquities given the lack of knowledge concerning the actual uranium enrichment

\section{Short-lived activities.}

The shori-lived impurities identified in these analyses are listed in Table III in decreasing order of their severity. Note that the concentrations are given in parts per million. Lanthanum can arise from the decay of Ba-140 in the fission of U-235 as well as from neutron activation of La-139. The lanthanum level (La-139) is determined as noted above in the previous section 

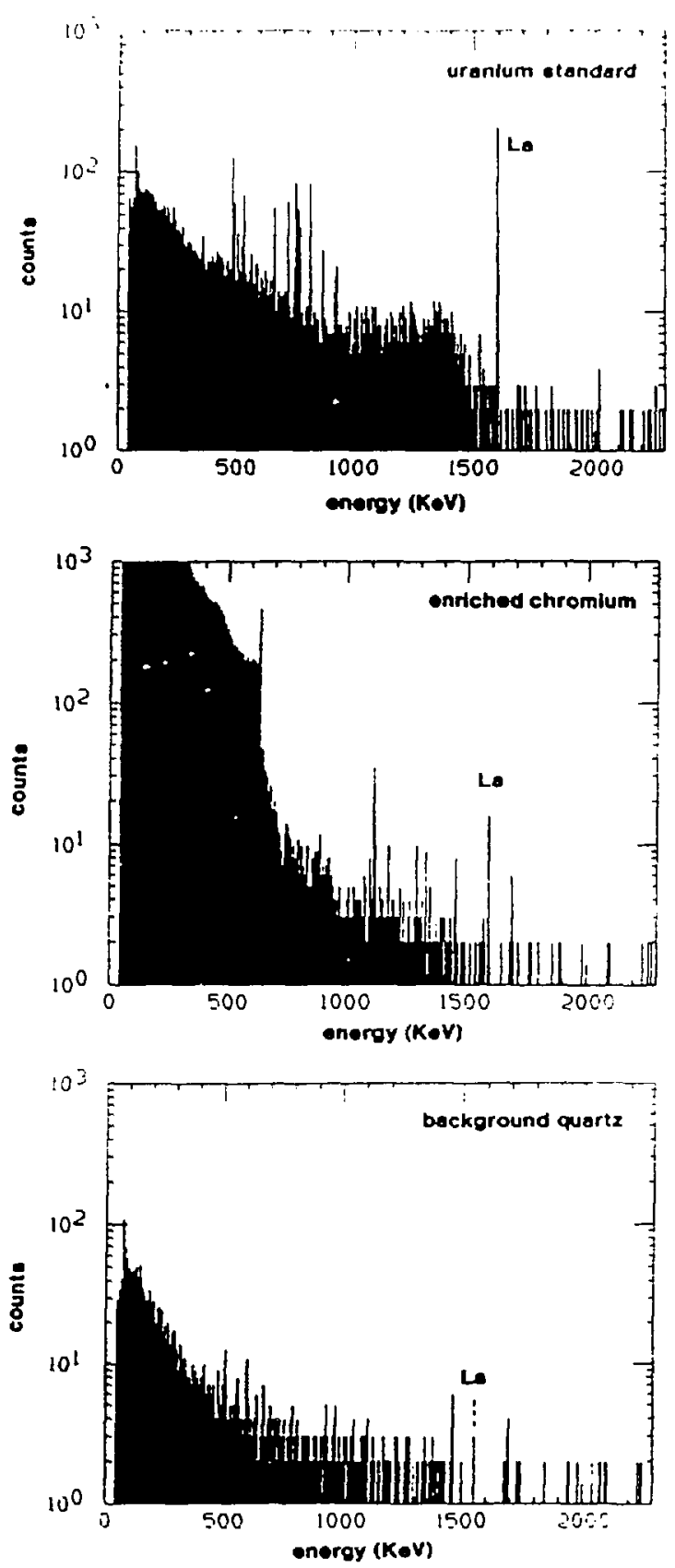

FIG. 10 . Gamma-ray spectra of the La-140 activity (1596 KeV as annotated) from fission produced $\mathrm{Ba}-140$ in (a) the uranium standard, (b) the enriched chromium test source and (c) the background quartz (where it is not evident). From this evidence and related analyses, uranium is found to be at the $2 \mathrm{ppm}$ level. The other peaks in the spectrum are either identified in the previous figures or are identified as fission products. The main point of the figure is to present the simplest evidence for establishing the uranium level based on direct comparison to a known standard. 
concerning the uranium contamination levels. The sodium levels are seen to be higher in the samples prepared from the chromyl-fluoride gas, and this aspect is further discussed below. The gallium and arsenic levels were near the threshold of detection, with positive results obtained for some samples and upper limits for other samples. Upper limits on the levels of copper are high since short-lived Cu-64 decays primarily by $\beta$-emission. Tungsten and potassium were additionally identified.

\section{Table III}

Summary of Detected Short-Lived Impurities in $\mathrm{Cr}_{2} \mathrm{O}_{3}$ Samples Produced from $\mathrm{CrO}_{2} \mathrm{~F}_{2}$ (in parts per million).

\begin{tabular}{lccccc}
\hline Element & $\begin{array}{c}\text { Nuclide/ } \\
\text { Half-life }\end{array}$ & $\begin{array}{c}\text { Enriched } \\
\text { Samples }\end{array}$ & $\begin{array}{c}\text { Leached } \\
\text { Samples }\end{array}$ & $\begin{array}{c}\text { ARC } \\
\text { Samples }\end{array}$ & $\begin{array}{c}\text { Chemical } \\
\text { Blank }\end{array}$ \\
\hline Lanthanum & La-139/1.7 d & $0.86 \pm 0.07$ & $0.53 \pm 0.08$ & $0.49 \pm 0.38$ & $0.51 \pm 0.11$ \\
Sodium & Na-24/0.625 d & $150 \pm 240$ & $170 \pm 150$ & $46 \pm 16$ & $16.2 \pm 2.5$ \\
Gallium $^{1}$ & Ga-72/0.59 d & $2.6 \pm 0.4$ & $\leq 1.0 \pm 0.3$ & $<0.4 \pm 0.10$ & $0.61 \pm 0.05$ \\
Arsenic ${ }^{1}$ & As-76/0.10 d & $0.41 \pm 0.22$ & $<0.40 \pm 0.09$ & $<0.65 \pm 0.14$ & $<0.50 \pm 0.11$ \\
Copper & Cu-64/0.53 d & $<26 \pm 1$ & $<570 \pm 130$ & $<18 \pm 4$ & $<15 \pm 3$ \\
Tungsten & W-187/0.996 d & $0.60 \pm 0.11$ & $0.32 \pm 0.04$ & $0.21 \pm 0.08$ & $0.22 \pm 0.10$ \\
Potassium & K-43/0.515d & $<21 \pm 1$ & $57 \pm 10$ & $20 \pm 6$ & $18 \pm 6$ \\
\hline N & & &
\end{tabular}

Note 1. Gallium and arsenic were detected in only some samples of a set as noted in Appendix C.

The main element of concern is sodium. Its neutron-activation product, $\mathrm{Na}-24$, decays with a $15 \mathrm{~h}$ half-life and a number of energetic gamma rays. Moreover, sodium is a common element. In the first series of runs the sodium levels were found to be high, at the thousands of ppm levels. Due to this discovery, 
a number of changes were made in the chemical procedure to eliminate any potential for the corrosive liquids to come into contact with metallic or glass surfaces. In the series of analyses described here, three samples each of a chemical blank and of natural chromium oxide were irradiated in addition to the three samples of enriched, leached and ARC, chromyl fluoride. These more detailed results are listed in Table IV below.

\section{Table IV}

Summary of Sodium Impurity Levels (in ppm).

\begin{tabular}{cccccc}
\hline Sample & $\begin{array}{c}\text { Chemical } \\
\text { Blank }\end{array}$ & $\begin{array}{c}\text { Enriched }^{2} \\
\mathrm{Cr}_{2} \mathrm{O}_{3}\end{array}$ & $\begin{array}{c}\text { Leached }^{2} \\
\mathrm{Cr}_{2} \mathrm{O}_{3}\end{array}$ & $\begin{array}{c}\mathrm{ARC}^{2} \\
\mathrm{Cr}_{2} \mathrm{O}_{3}\end{array}$ & $\mathrm{Cr}_{2} \mathrm{O}_{3}$ \\
& $18 \pm 4$ & $21 \pm 5$ & $120 \pm 20$ & Note 3 & $310 \pm 50$ \\
(a) & $13 \pm 5$ & $430 \pm 3$ & $340 \pm 50$ & $57 \pm 10$ & $780 \pm 120^{4}$ \\
(b) & $18 \pm 4$ & $2.5 \pm 2.3$ & $56 \pm 9$ & $35 \pm 6$ & $190 \pm 30$ \\
\hline
\end{tabular}

Note 1: $\mathrm{Cr}_{2} \mathrm{O}_{3}$ produced from $\mathrm{CrO}_{3}$.

Note 2: Produced from chromyl fluoride.

Note 3: The applicable spectrum was lost during file transfers; an estimate of $210 \pm 30 \mathrm{ppm}$ can be obtained from a longer duration count done for other purposes, which was not ideal for establishing shorter-lived activity levels.

Note 4: This sample, (b), was not fired in a furnace so that it could be compared to the other two samples (a) and (c), which were fired.

As can be judged from the results above, the chemical blank had fairly uniform sodium levels. These blanks should have identified any contamination problems in the procedures involving the wet chemistry. The chromyl-fluoride samples can be seen to have sodium levels which vary widely over two orders of 
magnitude. The chromium-oxide samples indicate a decrease of sodium upon firing (sample (b) vs. (a) and (c)).

\section{Possible Sources of Impurities.}

In the previous parts of this section (III.J) we discussed the long- and short-lived impurities detected in the chromium-oxide samples. A complete listing of the impurities is collected in Appendix $\mathrm{C}$ for the samples of interest. Where can these impurities come from?

A summary of the potential sources of the identified impurities is depicted in Table V. The first column lists the known inorganic fluoride gases, which are relevant to the work here, and their boiling points. The X's in the columns identify probable or potential sources of the impurity and the C's indicate sources of the impurity if corrosion occurs. The variability of the sodium levels could possibly depend on the atmospheric conditions, since sodium potentially could be picked up from the sea mist in the atmosphere at BNL (on the Eastern seaboard). 


\section{Table V}

\begin{tabular}{|c|c|c|c|c|c|}
\hline Element & $\begin{array}{l}\text { Fluoride } \\
\text { (boiling pt. } \\
\text { deg. C) }\end{array}$ & $\begin{array}{l}\text { Chromyl- } \\
\text { fluoride } \\
\text { gas }\end{array}$ & $\begin{array}{c}\text { Metal } \\
\text { system } \\
\text { and } \\
\text { handling }\end{array}$ & $\begin{array}{c}\text { Wet } \\
\text { Chemistry } \\
\text { Reactions }\end{array}$ & Remarks \\
\hline $\mathrm{Na}$ & no & & & $\mathrm{x}$ & $\begin{array}{l}\text { Variable } \\
\text { (potentially } \\
\text { atmospheric) }\end{array}$ \\
\hline $\mathrm{La}$ & no & & & $\mathbf{X}$ & \\
\hline $\mathrm{Ga}$ & no & & $\mathrm{C}$ & $\mathrm{X}$ & $\begin{array}{l}\text { Spike } \\
\text { (one sample) }\end{array}$ \\
\hline As & $\begin{array}{l}\mathrm{AsF}_{3}\left(-63^{\circ}\right) \\
\operatorname{AsF}_{5}\left(-53^{\circ}\right)\end{array}$ & $\mathrm{X}$ & $\mathrm{C}$ & & $\begin{array}{l}\text { Spike } \\
\text { (one sample) }\end{array}$ \\
\hline Zn & no & & $\mathrm{C}$ & $\mathrm{X}$ & \\
\hline $\mathrm{Fe}$ & no & & $\mathrm{C}$ & $\mathrm{X}$ & \\
\hline $\mathrm{Sc}$ & no & & & $\mathrm{X}$ & \\
\hline $\mathrm{Ta}$ & $\mathrm{TaF}_{8}\left(229.5^{\circ}\right)$ & $\mathrm{X}$ & & $\mathrm{X}$ & \\
\hline Sb & $\begin{array}{c}\mathrm{SbF}_{5}\left(145^{\circ}\right) \\
\mathrm{SbF}_{3}\left(319^{\circ} \text { sub }\right)\end{array}$ & $\mathrm{X}$ & & $\mathrm{X}$ & \\
\hline $\begin{array}{l}\text { Co } \\
\mathrm{U}\end{array}$ & $\begin{array}{c}\text { no } \\
\mathrm{UF}_{6}\left(56.2^{\circ}\right) \\
\end{array}$ & $\mathrm{X}$ & C & $\mathrm{X}$ & \\
\hline
\end{tabular}

$\mathrm{X}=$ Probable or potential source of impurity based on analysis.

$\mathrm{C}=$ Increased levels if corrosion occurs.

As noted earlier, in some of the chemical procedures, some samples were collected in a reaction vessel to which water was added in a closed system. After trying this approach, it was realized that the transfer of chromyl fluoride by cryogenic means is not always complete and some chromyl fluoride appears to coat the stainless-steel surfaces. This pinkish coating was observed to be stable when exposed to the atmosphere. A likely cause of this coating is either the reaction of chromyl fluoride with any surface water to form $\mathrm{CrO}_{3}$ or possibly some polymerization of $\mathrm{CrO}_{2} \mathrm{~F}_{2}$ on the surface. The pinkish material did not appear to 
have the red color characteristic of $\mathrm{CrO}_{3}$ but such judgments are clearly qualitative. On the other hand, chromyl fluoride has been reported to polymerize in the presence of UV light yielding a whitish material.

Regardless of the identity of the "chromyl fluoride-like" material which deposited on the metallic surfaces, in the presence of water vapor it can further react to form a corrosive chromic-acid solution. This was observed to have occurred in a number of samples produced during the second reactor run, and alluded to above in section III.E. Consequently these samples provide a good indication of the corrosion products expected from the stainless-steel tubing. Neutron activation analysis readily picked up high levels of iron, cobalt and copper. These samples were further analyzed using an electron microprobe. The $\mathrm{x}$-ray fluorescence spectrum typical of these samples is depicted in Fig. 11.

Nickel, which is more difficult to see using neutron activation, is readily evident in the $\mathrm{x}$-ray spectrum. Monel is basically a nickel-copper alloy whereas stainless steel has a higher iron and chromium content. Of all the potential impurities from corrosion, cobalt is perhaps the most severe and is more prevalent in stainless steels than in monel alloys. Regardless, the corrosion was easily avoided when we separated dry and wet steps in the procedure and utilized teflon sections, as described previously.

\section{Expected Radiation Rates and Limits in the 1-MCi Source.}

The activities found in the enriched chromium-oxide material produced in these test runs can be scaled up to examine the radioactive levels that would be expected in the 1-MCi $\mathrm{Cr}-51$ source. A number of considerations must be taken into account when attempting such a scale-up to $7 \mathrm{~kg}$ of enriched $\mathrm{Cr}-50$. The gamma-rays emitted from the central region of the source will interact with the source material itself leading to scattering and attenuation. In addition 
BNL MATERALS SCIzNCE8 DMANN

MON 26-FEB-90 15:19

Cursor: $0.000 \mathrm{keV}=0^{\circ}$

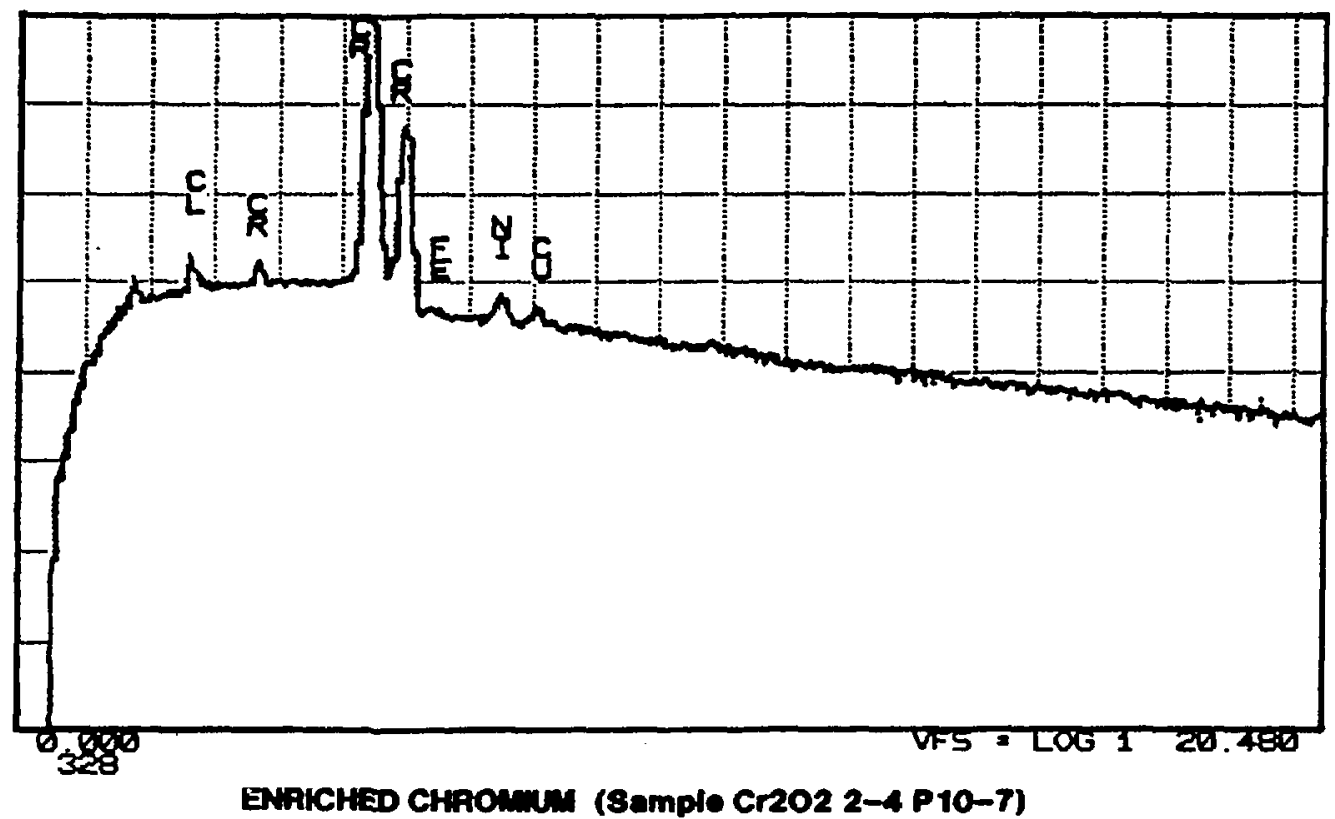

FIG. 11 Identified $\mathrm{Ni}, \mathrm{Cu}, \mathrm{Cl}$, Fe levels in enriched $\mathrm{Cr}$ material produced from chromyl fluoride contacting stainless steel in the presence of some water vapor. 
engineering considerations can be brought into play; especially in the design of the shielding. Consequently, the reduction in radiation levels outside the source can be achieved by design as well as by radiochemistry.

With these considerations aside, a worst case estimate can be made by simply treating the scaled-up source as a cylindrical source. Using the activity data $^{34}$ summarized in Appendix D, we can calculate the radiation levels at 2 meters from the cylindrical source, assuming the impurity levels found in the enriched chromium-oxide test samples. In this calculation, we also assume an irradiation flux of $7 \times 10^{13}$ neutrons $/ \mathrm{cm}^{2}$-sec over the continuous irradiation period of 21 days. Thus shorter-lived isotopes are produced mostly during the final days of the irradiation whereas the long-lived isotopes build up roughly linearly. We also assume $8.5 \mathrm{~cm}$ tungsten shielding (as now suggested in Ref. 14), approximate the attenuation as exponential and assume an overall $0.765 \mathrm{~cm}^{-1}$ average gamma-ray extinction coefficient characteristic of $2 \mathrm{MeV}$ gamma-rays in tungsten. These assumptions are conservative since the attenuation of gamma rays in tungsten has a weak energy dependence from 1 to $5 \mathrm{MeV}$, while the majority of gamma rays are less than $2 \mathrm{MeV}$ in energy. It is a currently assumed tenet of the GALLEX Collaboration that to reduce the flux in the final few days of the irradiation just to reduce the levels of the short-lived activities would be too high a cost given the resulting reduction of Cr-51 activity.

The presence of small amounts of impurities in the smaller samples could well remain as small amounts in much larger samples when the source size is scaled up, so the impurity levels, in ppm, could decrease significantly. The activity from impurities would be much reduced--that is, it is conservative to assume that each impurity concentration scales linearly.

At the end of the irradiation, the $1.08-\mathrm{MCi} \mathrm{Cr}$ source results in a trivially low $\left(<10^{-16} \mathrm{mr} / \mathrm{hr}\right)$ radiation level due to $\mathrm{Cr}-51$ at 2 meters from the source and 
outside the tungsten shield. The sodium activity is the highest of all impurities and stands apart in magnitude from all the other activities. At the activity level indicated in Fig. 12, the radiation level from Na-24 after 3 days is roughly 2 orders of magnitude above the $0.25 \mathrm{mr} / \mathrm{hr}$ limit, which is the health-safety exposure limitation which must be met while handling the source in France and Italy. The level of sodium is of concern, but the sodium amount that we assume in a scaled-up source could be overestimated.

In Fig. 12 we depict the radiation dose as a function of time due to each impurity. If we take the bench-mark level of $0.25 \mathrm{mr} / \mathrm{hr}$ at 2 meters from the cylindrical source as one constraint we must meet, (depicted by the horizontal line in the figure), we find that long-lived activities pose no problems. Moreover it would be expected that continued research and development would eliminate many of those listed impurities since there are no relevant volatile fluorides of zinc, iron or scandium. As seen graphically in Fig. 12, we have also drawn a vertical line at the 3 day point after the irradiation which, as a bench-mark, indicates the preferred day to transport the source from the reactor to the GALLEX site. As seen here, short-lived activities could pose a problem if not addressed further in the source design. Since it is anticipated that the "scale-up" procedure potentially overestimates the level of many of the indicated isotopes, the production of large amounts of chromium trioxide from chromyl fluoride will be the test that sodium levels are reduced in a large-scale operation.

It is worth pointing out that, based on measured impurity levels of sodium in commercially produced chromium, sodium levels may prove difficult to reduce to below the $1 \mathrm{ppm}$ level. If this is the case, then the three-day shipping requirement will have to be relaxed, the transport shielding may have to be increased or the source design may require more shielding. None of these requirements would be prohibitive. 


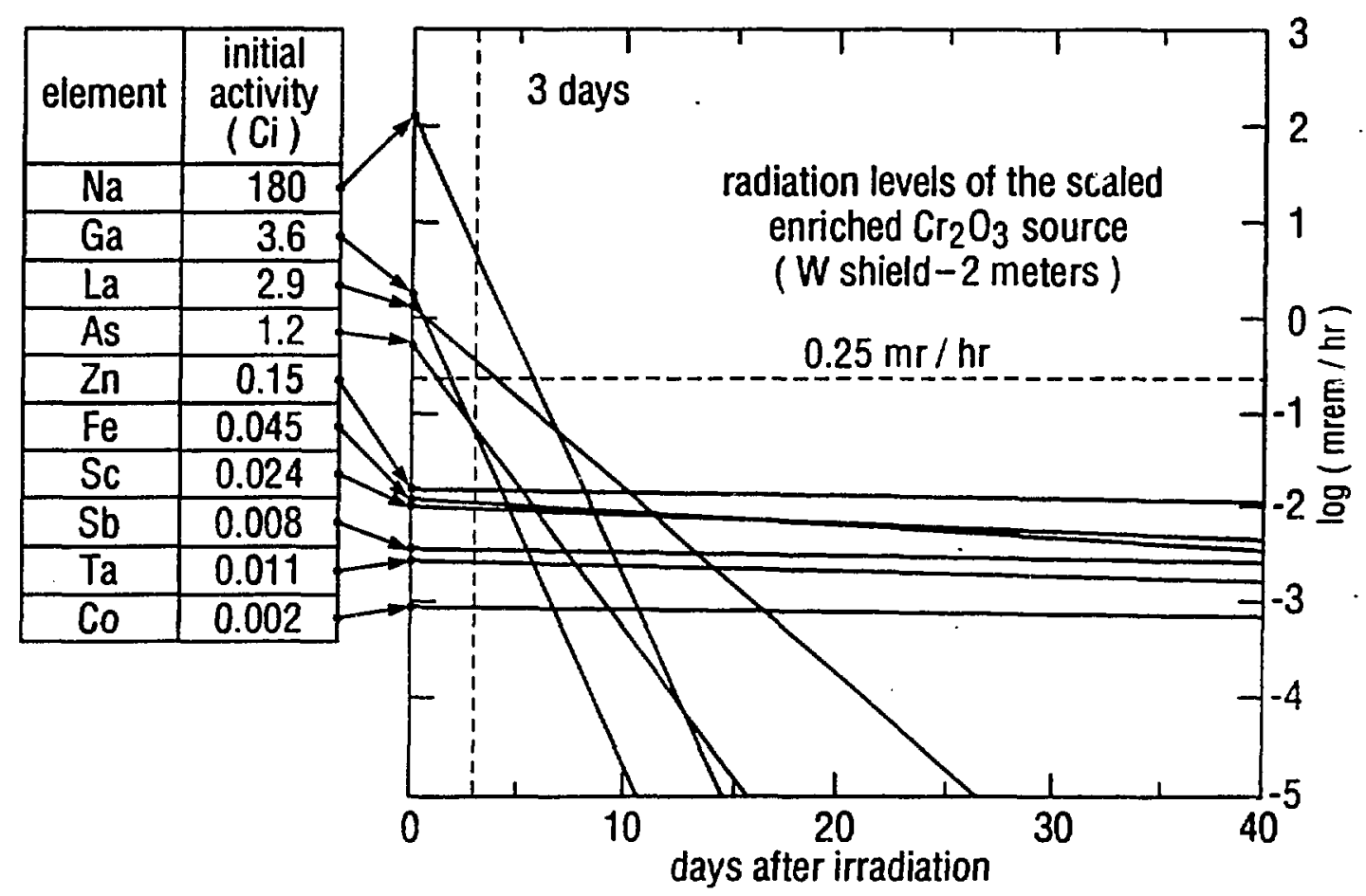

FIG. 12 Radiation dose due to impurities in the scaled up source. The long-lived activities are within the $0.25 \mathrm{mr} / \mathrm{hr}$ specification used as a guideline. The short-lived activities are slightly outside the three day guideline. It is expected that the scaled up source over-estimates contributions from such impurities. These radiation levels are the average of three samples of $\mathrm{Cr}_{2} \mathrm{O}_{3}$ produced from enriched chromyl fluoride. The levels are calculated at 2 meters from a cylindrical source having an $8.5 \mathrm{~cm}$ tungsten shield. 


\section{Summary of Radiation Levels from Chromium Tests.}

It is worthwhile to now compare the radioactive impurities of existing chromium metal grains available from a number of manufacturers as analyzed by NAA in a study by Katcoff ${ }^{35}$, along with the activities from an enriched source as discussed above and with the previously discussed large-scale chromium-grain test already carried out by GALLEX. ${ }^{13}$ The chromium-grain test is broken down into two samples for purposes of illustration: "a" refers to the grains as analyzed by the manufacturer and " $b$ " refers to the grains as analyzed by the Orsay-LMEI group. As seen in Fig. 13, the activities from each of these sources were binned into two groups, either short-lived or long-lived activites, to simplify the analyses. The $x$-component of each plotted point indicates the radiation levels due to the short-lived activities and the y-component the radiation levels due to the long-lived activities. For the purposes of illustration, the radiation levels are those on contact with the tungsten shield at 3 days after the irradiation.

The most striking feature evident from the plot in Fig. 13 is the high levels of the short-lived radiations. The major contributor to all of these high levels is the sodium content. A second feature is also clearly evident. Fig. 13 indicates that few source materials, namely the enriched sample and one metal grain, meet the long-lived activity limit. What one can argue from this picture is that the production of any enriched-chromium source from chromyl fluoride, be it ultimately produced in the form of a chromium metallic particle or in the form of chromium oxide, has potential advantages in producing a source material very clean of long-lived impurities. The short-lived impurity questions remained to be resolved.

From our tests we learn that the levels of short-lived activities exceed the specifications indicated in Ref. 13 both for radiation levels on contact with the 
Calculated Badiation Levels (W-shield, on contact, 3 days post-irradiation )

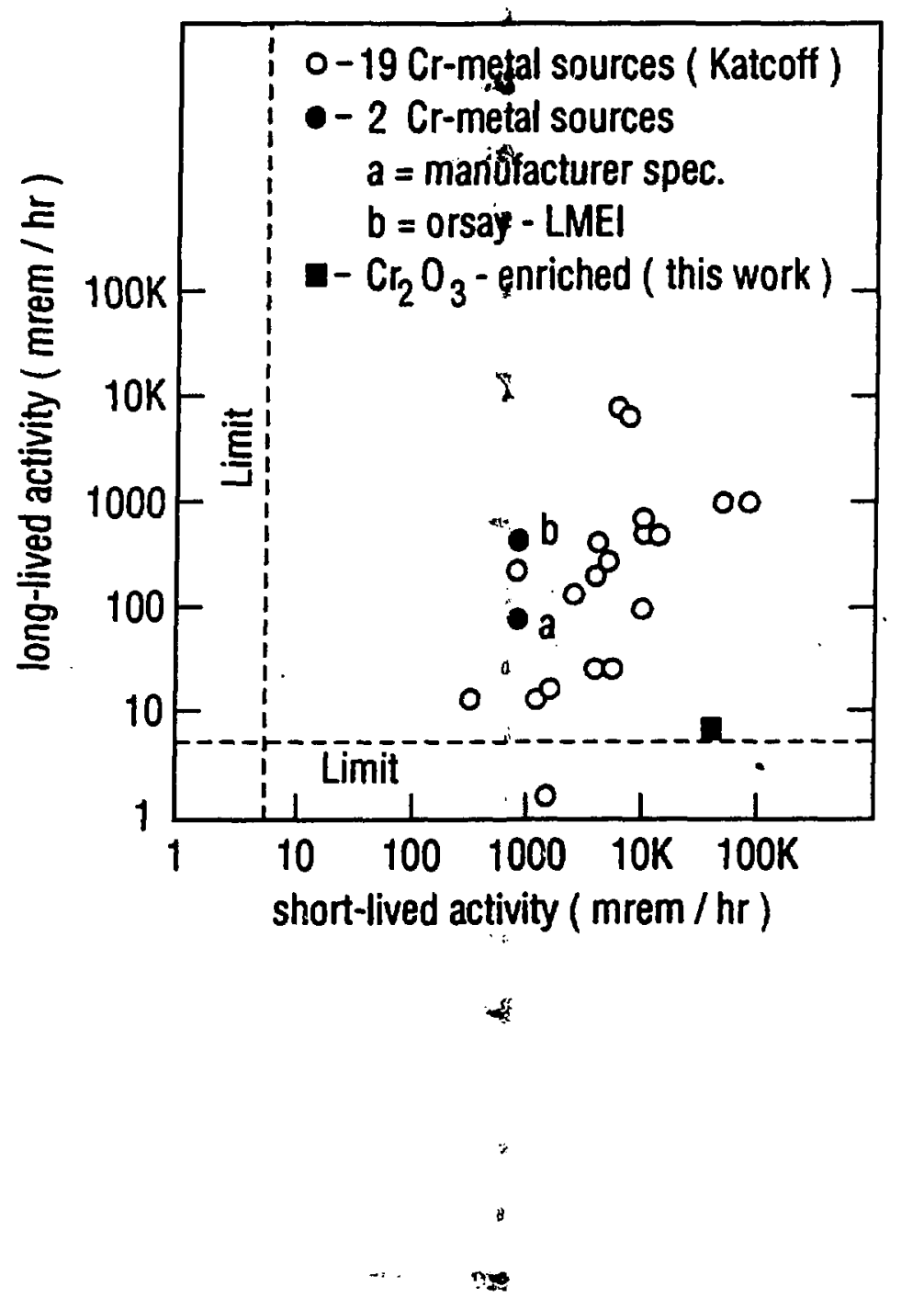

FIG. 13 Radiation-level estimates for various sources on contact with a tungsten shield at 3 days after irradiation. The values for the nineteen $\mathrm{Cr}$-metal sources are based on impurity levels of various purchased grains, and the two points $a$ and $b$ depict levels from the GALLEX grain test (Ref. 13). The enriched source (an average of the three test sources scaled-up), has too high a level of short-lived activities. The limits indicated in the figure are the currently accepted guidelines for the radiation level on contact with the shield $(6 \mathrm{mrem} / \mathrm{hr})$. 
shield and at 2 meters away from it. The short-lived level is about 20 to 50 times higher than the specification of $0.25 \mathrm{mrem} / \mathrm{hr}$ at 2 meters at a time 3 days after the irradiation. The levels on contact, though, are much worse; for example, the activity level of the short-lived component is about a factor of $10^{4}$ too high. Since this basic problem is common to all chromium materials to date, we do not address its resolution here. How and by how much we need to reduce the sodium levels in the final source depend on its ultimate form, preparation and shielding design. Final acceptable impurity limits can be set with further refinements of the source design.

\section{Conclusions}

We have produced and irradiated enriched $\mathrm{Cr}-50$ sources and studied their properties as preparation for the production of an intense mega-curie neutrino-source for tests of the GALLEX detector. The source examined here is an enriched source in the form of chromium-oxide. Previous work has been reported on a natural chromium source in the form of metallic particles (Ref. 13) and new work is in progress examining an enriched source of chromium metal particles made by electrolysis (Ref. 14). The results of this and the previous study reiniorce the intent of GALLEX to make a chromium source instead of the suggested argon gas source, (Ref. 15).

The results of this experimental work document the feasibility of enriching chromium in $\mathrm{Cr}-50$ by gas centrifugation of chromyl fluoride and subsequently converting it to a form suitable for reactor irradiation. The ultimate choice of metal or chromium oxide powder is thus relegated to engineering design considerations. The procedures developed herein are also applicable to impurity tests of chromyl fluoride samples obtained from future gas-centrifuge tests. 
In summary, then:

1. Chromyl fluoride conversion to the oxide for analysis or as the final source material was developed.

2. Neutron irradiation results of chromyl fluoride samples, in particular, $21.4 \%$ enriched $\mathrm{Cr}-50$ chromyl fluoride, revealed no severely limiting long-lived impurities.

3. Short-lived activities are a concern in any chromium source.

Finally, it is necessary to address the materials properties of the chromium oxide. Whereas the chromium metal can be conveniently produced as large ( $\mathrm{mm}$ size) grains, chromium oxide might prove more difficult to produce in such sizes. A report on the study of chromium materials is in preparation. ${ }^{24}$ 


\section{Acknowledgement.}

The authors of this report acknowledge very helpful discussions on various aspects of these investigations, in part due to the cross-disciplinary nature of some of the work involved. We thank Garman Harbottle for discussions of neutron activation techniques, Seymour Katcoff for discussions and information concerning the irradiation of chromium metal particles and Walter Kunnmann for numerous discussions on chromium crystal properties, chromium-vanadium materials and for the use of some of his furnaces, and Robert Sabatini for work using the scanning-electron microscope and microprobe analysis. We appreciate discussions with the other members of the GALLEX collaboration, most notably Michel Cribier, the assistance of the reactor groups at BNL and the collaboration with A. Szady, W. L. Roberts, and C. Liles at the gas-centrifuge facility at ORNL. 


\section{References}

1. R. Davis, K. Lande, B. T. Cleveland, J. Ullman and J. K. Rowley, Neutrino '88, Boston, World Scientific, 518 (1989).

2. K. S. Hirata et. al. (Kamiokande-II Collaboration), Phys. Rev. Lett. 63, 16 (1989); Phys. Rev. Lett. 65, 1297 (1990).

3. S. Turck-Chieze, S. Cahan, M. Casse and C. Doom, Astrophysical Journal 335,415 (1988).

4. J. N. Bahcall, R. K. Ulrich, Rev. Mod. Phys. 60, 297 (1988).

5. M. Cribier and the GALLEX Collaboration, "The Status of GALLEX", submitted to the 25th International Conference on High Energy Physics, Singapore, August 2-8, 1990.

6. V. Gavrin, Proceedings of the IAU Colloquium No. 121, Inside the Sun, edited by G. Berthomieu and M. Cribier, Kluwer Academic Publ., p. 187 (1990), and Proceedings of the Conference on Neutrinos '90 (to be published).

7. A. J. Baltz and J. Weneser, Phys. Rev. D 37,3364 (1988).

8. S. Mikheyev and A. Smirnov, Progress in Particle and Nuclear Physics, 23, 41 (1989).

9. L. Wolfenstein, Phys. Lett. B 194, 197 (1987).

10. T. K. Kuo and J. Pantaleone, Rev. Mod. Phys. 61, 937 (1989).

11. J. N. Bahcall and H. A. Bethe, Phys. Rev. Lett. 65, 2233 (1990).

12. I. R. Barabanov et. al., "Pilot Installation of the Gallium-Germanium Solar Neutrino Telescope", in Solar Neutrinos and Neutrino Astronomy, AIP Conference Proceedings No. 126, edited by M. L. Cherry, K. Lande and W. A. Fowler, (AIP,New York, 1985) p 175.

13. M. Cribier, et al., Nucl. Inst. and Methods in Phys. Res. A265, 574 (1988). 
14. M. Cribier, "Electrolysis", presentation at the XII Gallex Workshop 1990 (unpublished).

15. W. Haxton, Phys. Rev. C 38,2474 (1988).

16. R. S. Raghavan, Neutrino-78, Brookhaven (unpublished, cited in Ref. 12).

17. L. W. Alvarez, Physics Notes, Lawrence Radiation Lab, 1973 (cited in Ref. 12).

18. W. Hampel, in the Proceedings of the 7 th International Conference on Atomic Masses and Fundamental Constants (AMCO-7), Darmstadt-Seeheim, F. R. Germany, 3-7 Sep. 1984.

19. W. Hampel, "The Gallium Solar Neutrino Experiment" in Solar Neutrinos and Neutrino Astronomy, AIP Conference Proceedings No. 126, eds. M. L. Cherry, W. A. Fowler and K. Lande, (AIP, New York, 1985), p. 162.

20. W. Hampel and L. Remsberg, Phys. Rev. C 31, 666 (1985).

21. D. Krofcheck et. al. Phys. Rev. Letts. $\underline{55}, 1051$ (1985).

22. Orihara et. al., Phys. Rev. Letts. 51, 1328 (1983).

23. T. Baltz, J. Weneser, B. Brown and J. Rapaport, Phys. Rev. Letts. $\underline{53}, 2078$ (1984).

24. F. X. Hartmann, BNL Report (in preparation).

25. W. Kwasnik, "Chromyl Fluoride" in Handbook of Preparative Inorganic Chemistry Vol. 1 Second. Ed., edited by George Brauer (Academic Press, New York, 1963) pp.258-259.

26. A. Engelbrecht and A. V. Grosse, J. Am. Chem. Soc. 74, 5262 (1952).

27. A. J. Szady, Nucl. Inst. and Methods in Phys. Research A282, 277 (1989).

28. F. A. Cotton and G. Wilkinson, Advanced Inorganic Chemistry, 3rd ed. (John Wiley and Sons, New York, 1972). (See also Gmelin, Vol. 52, p.145.)

29. Handbook of Chemistry and Physics, The Chemical Rubber Company, 1989. 
30. W. W. Scott, Standard Methods of Chemical Analysis, 5th Ed. edited by N. Howell Furman (D. Van Nostrand Company, Inc., New York, 1939).

31. The Intral code used here is a modified version of the code modified previously by J.B. Cumming (unpublished) combined with a modified version of an INRAL control code by G. Harbottle (unpublished). The original version is by Gunnick, Levy, and Niday, U. of Ca. Rad. Lab. Report No. UCID-15140 (1976) (unpublished). In our work, a computer program, called CRANAL takes the modified INTRAL output and produces the impurity analyses for chromium samples.

32. William S. Lyon, Jr., editor, Guide to Activation Analysis, (D. Van Nostrand Company, Inc. Princeton, 1964).

33. A. Szady, ORNL (private communication) at the Gallex Workshop, April 1988 (unpublished).

34. Radiological Health (Rev, Edition), Public Health Service, U.S. Dept. of Health, Education and Welfare, U.S. Government, Jan. 1970.

35. S. Katoff, "Sensitive Neutron Activation Method for Determination of Trace Impurities in Cr-Metal," in Fifth International Conference on Nuclear Methods in Environmental and Energy Research, Mayaquez, Puerto Rico, April 2-6, 1984. 
Appendix A. Summary of Irradiated Samples.*

Irradiation 1. Standards and Procedural Checks.

\begin{tabular}{|c|c|c|c|c|c|}
\hline No. & Sample & No. & Sample & No. & Sample \\
\hline 1 & $\mathrm{Cr}_{2} \mathrm{O}_{3}$ (shelf-A) & 7 & $\mathrm{Cr}_{2} \mathrm{O}_{3}$ (from $\left.\mathrm{CrO}_{3}\right)$ & 13 & Fe standard \\
\hline 2 & $\mathrm{Cr}_{2} \mathrm{O}_{3}$ (shelf-B) & 8 & $\mathrm{Cr}_{2} \mathrm{O}_{3}$ (from $\left.\mathrm{CrO}_{3}\right)$ & 14 & $\begin{array}{l}\text { Cu standard } \\
\text { (not irradiated) }\end{array}$ \\
\hline 3 & $\mathrm{Cr}_{2} \mathrm{O}_{3}$ (shelf-C) & 9 & Ag standard & 15 & U standard \\
\hline 4 & A - fired & 10 & Blank & 16 & $\begin{array}{l}\text { NBS standard } \\
\text { SRM-81 }\end{array}$ \\
\hline 5 & B - fired & 11 & Fe standard & 17 & Fe standard \\
\hline 6 & C - fired & 12 & $\mathrm{Na}, \mathrm{W}$ standard & & \\
\hline
\end{tabular}

Irradiation 2. First Chromyl Fluoride Analyses.

\begin{tabular}{ll|cl|ll}
\hline No. & $\begin{array}{l}\text { Sample } \\
1\end{array}$ & $\frac{\mathrm{No}}{\mathrm{Cr}_{2} \mathrm{O}_{3}(1-1)}$ & $\begin{array}{l}\text { Sample. } \\
\mathrm{Cr}_{2} \mathrm{O}_{3}(2-4)\end{array}$ & $\begin{array}{l}\text { No. } \\
13\end{array}$ & $\begin{array}{l}\text { Sample } \\
\mathrm{Cr}_{2} \mathrm{O}_{3} \text { (from } \\
\text { shelf } \mathrm{CrO} \text { ) }\end{array}$ \\
2 & $\mathrm{Cr}_{2} \mathrm{O}_{3}(2-1)$ & 8 & $\mathrm{Cr}_{2} \mathrm{O}_{3}(3-1)$ & .14 & $\begin{array}{l}\text { Cr-standard } \\
\text { crystal }\end{array}$ \\
3 & $\mathrm{Cr}_{2} \mathrm{O}_{3}(2-2)$ & 9 & $\mathrm{Cr}_{2} \mathrm{O}_{3}(3-2)$ & 15 & Blank \\
4 & $\mathrm{Cr}_{2} \mathrm{O}_{3}(1-2)$ & 10 & $\mathrm{Cr}_{2} \mathrm{O}_{3}(3-3)$ & 16 & $\mathrm{Na}, \mathrm{W}$ standard \\
5 & $\mathrm{Cr}_{2} \mathrm{O}_{3}(1-3)$ & 11 & $\mathrm{Fe}$ standard & & \\
6 & $\mathrm{Cr}_{2} \mathrm{O}_{3}(2-3)$ & 12 & $\mathrm{U}$ standard & & \\
\hline
\end{tabular}

Irradiation 3. Chromyl Fluoride Analysis Test.

No. Sample

$1 \quad \mathrm{Cr}_{2} \mathrm{O}_{3}$ (from

$\mathrm{CrO}_{2} \mathrm{~F}_{2}$ )

2 Blank

$3 \quad \mathrm{Cr}_{2} \mathrm{O}_{3}$ (from shelf $\mathrm{CrO}_{3}$ ) 
Irradiation 4. Second Chromyl Fluoride Analyses.

\begin{tabular}{|c|c|c|c|c|c|}
\hline No. & Sample & No. & Sample. & $\underline{N_{0}}$ & Sample \\
\hline 1 & $\mathrm{Cr}_{2} \mathrm{O}_{3}(1-1 \mathrm{~B})$ & 8 & $\mathrm{Cr}_{2} \mathrm{O}_{3}(2-3 \mathrm{~B})$ & 15 & $\mathrm{Na}, \mathrm{W}$ standard \\
\hline 2 & $\mathrm{Cr}_{2} \mathrm{O}_{3}(1-2 \mathrm{~B})$ & 9 & $\mathrm{Cr}_{2} \mathrm{O}_{3}$ (from $\mathrm{CrO}_{3}$ ) & 16 & $\mathrm{Cr}_{2} \mathrm{O}_{3}$ (shelf) \\
\hline 3 & $\mathrm{Cr}_{2} \mathrm{O}_{3}(1-3 \mathrm{~B})$ & 10 & $\mathrm{Cr}_{2} \mathrm{O}_{3}$ (from $\mathrm{CrO}_{3}$ ) & 17 & $\begin{array}{l}\mathrm{Cr}_{2} \mathrm{O}_{3} \\
\text { (shelf,fired) }\end{array}$ \\
\hline 4 & $\begin{array}{l}\mathrm{Cr}_{2} \mathrm{O}_{3} \\
\mathrm{CrO}_{3} \text { (from }\end{array}$ & 11 & Blank & 18 & $\mathrm{Cr}_{2} \mathrm{O}_{3}(\mathrm{ARC}-1)$ \\
\hline 5 & $\begin{array}{l}\mathrm{Cr}_{2} \mathrm{O}_{3} \\
\text { (shelf,fired) }\end{array}$ & 12 & Blank & 19 & $\mathrm{Cr}_{2} \mathrm{O}_{3}(\mathrm{ARC}-2)$ \\
\hline 6 & $\mathrm{Cr}_{2} \mathrm{O}_{3}(2-1 \mathrm{~B})$ & 13 & U standard & 20 & $\mathrm{Cr}_{2} \mathrm{O}_{3}(\mathrm{ARC}-3)$ \\
\hline 7 & $\mathrm{Cr}_{2} \mathrm{O}_{3}(2-2 \mathrm{~B})$ & 14 & $\mathrm{Na}, \mathrm{W}$ Standard & & \\
\hline
\end{tabular}

*Legend: As noted in the text in Section III, Samples labelled by an initial "1-" come from "leaching" $\mathrm{CrO}_{2} \mathrm{~F}_{2}$; "2-"come from enriched $\mathrm{CrO}_{2} \mathrm{~F}_{2}$; "3-" come from "manufacturers" $\mathrm{CrO}_{2} \mathrm{~F}_{2}$ gas and "ARC-" come from ARC chromyl fluoride gas. Three shelf sources labelled $\mathrm{A}, \mathrm{B}, \mathrm{C}$ were used along with $\mathrm{Cr}_{2} \mathrm{O}_{3}$ from shelf $\mathrm{CrO}_{3}$ as a "chemical blank". The "shelf" material is reagant grade. Blanks in the listings refer to empty quartz ampoules. 
Appendix B. Nuclear Data Relevant to Chromium Impurity Analysesa

\begin{tabular}{|c|c|c|c|c|c|c|}
\hline Element & Z & Nuclide & $\begin{array}{l}\text { Gamma-Ray } \\
\text { Energy } \\
(\mathrm{MeV})\end{array}$ & $\begin{array}{l}\gamma \text { 's per } 100 \\
\text { decays }\end{array}$ & $\begin{array}{l}\text { Effective } \\
\text { Cross-Sectionb } \\
\text { (barns) }\end{array}$ & Half-life \\
\hline Sodium & 11 & $\mathrm{Na}-24$ & $\begin{array}{l}1368.550 \\
1733 \\
2244\end{array}$ & $\begin{array}{l}100.00 \\
100 \\
100\end{array}$ & 0.53 & $15.03 \mathrm{~h}$ \\
\hline Silicon & 14 & Si-30 & 1266.1 & 0.07 & 0.033 & $2.62 \mathrm{~h}$ \\
\hline Potassium & 19 & $\begin{array}{l}K-40 \\
K-42\end{array}$ & $\begin{array}{l}1460.750 \\
1524.7\end{array}$ & $\begin{array}{l}10.70 \\
17.9\end{array}$ & $\begin{array}{l}1.96 \\
0.098\end{array}$ & $\begin{array}{l}1.28 \times 10^{9 y} \\
12.36 \mathrm{~h}\end{array}$ \\
\hline Calcium & 20 & $\mathrm{Ca}-47$ & 1297 & 77 & 0.000028 & $4.54 \mathrm{~d}$ \\
\hline Scandium & 21 & $\mathrm{Sc}-46$ & $\begin{array}{r}889.260 \\
1120.520\end{array}$ & $\begin{array}{l}99.98 \\
99.99\end{array}$ & 26.5 & $83.85 \mathrm{~d}$ \\
\hline Chromium & 24 & $\mathrm{Cr}-51$ & 320.070 & 10. & 0.69 & $27.7 \mathrm{~d}$ \\
\hline Manganese & 25 & $\mathrm{Mn}-56$ & $\begin{array}{l}1811.2 \\
2112.6\end{array}$ & $\begin{array}{l}27.2 \\
14.3\end{array}$ & 13.3 & $2.57 \mathrm{~h}$ \\
\hline Iron & 26 & $\mathrm{Fe}-59$ & $\begin{array}{r}142.650 \\
192.340 \\
1099.220 \\
1291.56\end{array}$ & $\begin{array}{r}1.03 \\
3.11 \\
56.50 \\
43.20\end{array}$ & 0.0036 & $45.1 \mathrm{~d}$ \\
\hline Cobalt & 27 & $\mathrm{Co}-60$ & $\begin{array}{l}1173.20 \\
1332.51\end{array}$ & $\begin{array}{l}99.86 \\
99.98\end{array}$ & 37 & $5.27 \mathrm{y}$ \\
\hline Copper & 29 & $\mathrm{Cu}-64$ & 1346 & 0.6 & 3.1 & $12.7 \mathrm{~h}$ \\
\hline Zinc & 30 & $\mathrm{Zn}-65$ & 1116 & 51 & 0.38 & 244. d \\
\hline Gallium & 31 & $\mathrm{Ga}-72$ & $\begin{array}{r}600.9 \\
629.9 \\
834.0 \\
894.2 \\
1050.7 \\
1596.7 \\
1710.9 \\
2201.7\end{array}$ & $\begin{array}{c}5.58 \\
25.2 \\
95.6 \\
9.85 \\
6.92 \\
4.23 \\
0.39 \\
25.6\end{array}$ & 1.88 & $13.95 \mathrm{~h}$ \\
\hline Arsenic & 33 & As -76 & $\begin{array}{l}1216 \\
1229 \\
\end{array}$ & $\begin{array}{l}3.8 \\
1.4 \\
\end{array}$ & 4.3 & $26.3 \mathrm{~h}$ \\
\hline
\end{tabular}


Appendix B. continued

\begin{tabular}{|c|c|c|c|c|c|c|}
\hline Element & Z & Nuclide & $\begin{array}{l}\text { Gamma-Ray } \\
\text { Energy } \\
(\mathrm{MeV})\end{array}$ & $\begin{array}{l}\gamma \text { 's per } 100 \\
\text { decays }\end{array}$ & $\begin{array}{l}\text { Effective } \\
\text { Cross-Sectionb } \\
\text { (barns) }\end{array}$ & Half-life \\
\hline Bromine & 35 & $\mathrm{Br}-82$ & $\begin{array}{l}1317 \\
1475\end{array}$ & $\begin{array}{l}27 \\
17\end{array}$ & 1.33 & $35.3 \mathrm{~h}$ \\
\hline Rubidium & 37 & $\mathrm{Rb}-86$ & 1076.6 & 8.76 & 0.29 & $18.63 \mathrm{~d}$ \\
\hline Silver & 47 & $\mathrm{Ag}-110$ & $\begin{array}{r}885 \\
937 \\
1384\end{array}$ & $\begin{array}{l}73 \\
34 \\
24\end{array}$ & 2.2 & $252 \mathrm{~d}$ \\
\hline Tin & 50 & $\mathrm{Sn}-125$ & $\begin{array}{l}2001.7 \\
1066.6\end{array}$ & $\begin{array}{l}2.13 \\
8.86\end{array}$ & 0.0002 & $9.63 \mathrm{y}$ \\
\hline Antimony & 51 & $\mathrm{Sb}-124$ & $\begin{array}{l}1691 \\
2091\end{array}$ & $\begin{array}{r}49 \\
6\end{array}$ & 1.84 & $60 \mathrm{~d}$ \\
\hline Tellurium & 52 & Te-131 & $\begin{array}{r}773.7 \\
852.2 \\
1125.5 \\
2000.9\end{array}$ & $\begin{array}{l}38.1 \\
21 \\
11.4 \\
2.01\end{array}$ & 0.00676 & $32.4 \mathrm{~h}$ \\
\hline Cesium & 55 & Cs-134 & $\begin{array}{r}604.7 \\
795.8 \\
1038.5 \\
1167.9 \\
1365.1\end{array}$ & $\begin{array}{c}97.6 \\
85.4 \\
1 \\
1.8 \\
3.04\end{array}$ & 29.6 & $2.06 \mathrm{y}$ \\
\hline $\begin{array}{l}\text { Lantha- } \\
\text { num }\end{array}$ & 57 & La-140 & $\begin{array}{l}328.75 \\
487.030 \\
\mathbf{8 1 5 . 8} \\
867.8 \\
925.250 \\
1596.2\end{array}$ & $\begin{array}{c}18.50 \\
43.00 \\
22.32 \\
5.35 \\
6.92 \\
95.5\end{array}$ & 9 & $40.27 \mathrm{~h}$ \\
\hline Europium & 63 & Eu-152 & $\begin{array}{c}121.80 \\
344.3 \\
778.9 \\
964 \\
1085.8 \\
1112.070 \\
1408.08\end{array}$ & $\begin{array}{l}29.24 \\
27 \\
12.99 \\
14.58 \\
10.29 \\
13.58 \\
21.21\end{array}$ & 1577 & $12.7 \mathrm{y}$ \\
\hline Terbium & 65 & $\mathrm{~Tb}-160$ & $\begin{array}{r}966.2 \\
1177.9\end{array}$ & $\begin{array}{l}25.5 \\
15.5\end{array}$ & 23 & $72.4 \mathrm{~d}$ \\
\hline Holmium & 67 & $\begin{array}{l}\text { Ho-166 } \\
\text { Ho-166m }\end{array}$ & $\begin{array}{c}80.57 \\
1379.4 \\
80.57\end{array}$ & $\begin{array}{l}6.2 \\
0.93 \\
12.5\end{array}$ & $\begin{array}{l}62 \\
3.5 \\
\end{array}$ & $\begin{array}{r}26.8 \mathrm{~h} \\
1200 \mathrm{y} \\
\end{array}$ \\
\hline
\end{tabular}


Appendix B. continued

\begin{tabular}{|c|c|c|c|c|c|c|}
\hline Element & Z & Nuclide & $\begin{array}{l}\text { Gamma-Ray } \\
\text { Energy } \\
(\mathrm{MeV})\end{array}$ & $\begin{array}{l}\gamma \text { 's per } 100 \\
\text { decays }\end{array}$ & $\begin{array}{l}\text { Effective } \\
\text { Cross-Sectionb } \\
\text { (barns) }\end{array}$ & Half-life \\
\hline Thulium & 69 & $\mathrm{Tm}-170$ & 84.262 & 3.2 & 105 & $128.6 \mathrm{~d}$ \\
\hline Tantalum & 73 & Ta-182 & $\begin{array}{l}1189 \\
1221\end{array}$ & $\begin{array}{l}16 \\
27\end{array}$ & 21 & $115 \mathrm{~d}$ \\
\hline Tungsten & 74 & W-187 & $\begin{array}{r}72.5 \\
134.2 \\
479.5 \\
551.6 \\
618.4 \\
625.5 \\
685.8 \\
772.9\end{array}$ & $\begin{array}{c}12.9 \\
10.3 \\
25.3 \\
5.89 \\
7.27 \\
1.26 \\
31.6 \\
4.77\end{array}$ & 10.9 & $23.9 \mathrm{~h}$ \\
\hline Iridium & 77 & Ir-192 & $\begin{array}{l}316.5 \\
468.1\end{array}$ & $\begin{array}{l}82.9 \\
48.1\end{array}$ & 116 & $74 \mathrm{~d}$ \\
\hline Gold & 79 & $\mathrm{Au}-198$ & 411.8 & 95.5 & 88.2 & $2.7 \mathrm{~d}$ \\
\hline \multirow[t]{2}{*}{ Uranium } & 92 & $\mathrm{Te}-132^{\mathrm{c}}$ & $\begin{array}{l}228.2 \\
773 \\
667.7\end{array}$ & $\begin{array}{l}88 \\
79 \\
98.7\end{array}$ & 0.28 & $78 d$ \\
\hline & & $\mathrm{Ba}-140^{c}$ & $\begin{array}{r}487 \\
537.3 \\
1596.2 \\
\end{array}$ & $\begin{array}{l}49.4 \\
19.9 \\
96 \\
\end{array}$ & 0.26 & $12.8 \mathrm{~d}$ \\
\hline
\end{tabular}

a This listing is of practical use in the analysis of chromium material impurities. It is not an all-inclusive listing of all gamma rays. It serves as a ready reference of useful gamma-rays and isotopes which characterize a given element.

b Effective cross-section $=(n, \gamma)$ cross-section multiplied by the fractional abundance of the target isotope.

c From thermal-neutron fission of U-235. 
Appendix C. Complete Listing of Analyzed Impurities in $\mathrm{Cr}_{2} \mathrm{O}_{3}$ Samples (ppm).

$\mathrm{CrO}_{3} \quad$ Enriched Leached ARC

Eu

$$
\begin{array}{rr}
\leq 0.0070^{2 \gamma} & \leq 0.0072^{1 \gamma} \\
\pm 0.0026 & \pm 0.0017
\end{array}
$$

0.0062

$<0.0017$

Sc

0.020

0.0049

$$
\pm 0.0003
$$

0.014

$\pm 0.0013$ $\pm 0.0003$

Ag $\quad<0.038$

$$
\pm 0.007
$$

$<0.18$

$$
\pm 0.02
$$

$<0.038$

$\pm 0.007$

0.0064

$\pm 0.0002$

Co

0.054 $\pm 0.004$

0.0076 $\pm 0.0063$

0.065

$$
\pm 0.004
$$

$0.034^{1 \gamma}$

$$
\pm 0.016
$$

$\mathrm{Sb}$

$$
\begin{aligned}
& 0.20 \\
& \pm 0.01
\end{aligned}
$$

0.049

$\mathrm{Ta}$

0.0075

$$
\pm 0.0018
$$

0.016

$$
\pm 0.018
$$

0.20

$$
\pm 0.03
$$

0.037

$$
\pm 0.003
$$

0.091

$$
\pm 0.006
$$

U $\quad 0.89$

$$
\pm 0.05 \quad 2.0 \pm 0.04
$$

0.015

$$
\pm 0.002
$$

0.0085

$$
\pm 0.0017
$$

0.16

$$
\pm 0.02
$$

0.49

$$
\pm 0.03
$$

$\mathrm{Tb}$

$$
\leq 0.0072^{1 \gamma}
$$

$$
\pm 0.0020
$$

$<0.011$

$$
\pm 0.001
$$

$<0.0027$

$\pm 0.0005$

$<0.0025$

Ir

$$
\begin{aligned}
& <0.00037 \\
& \quad \pm 0.00007
\end{aligned}
$$

$$
<0.0015
$$$$
\pm 0.0002
$$

$$
25 \pm 3(1 \gamma, 1 \mathrm{~s})
$$

$\pm 0.0005$

Cs

$$
\leq 0.0201 \gamma
$$

$<0.012$

$$
\pm 0.0013
$$

$\leq 0.030^{1 \gamma}$

$$
\pm 0.004
$$$$
0.014^{1 \gamma}
$$

$\mathrm{Te}$

$$
<44 \pm 10
$$$$
<110 \pm 10
$$$$
<36 \pm 8
$$$$
<58 \pm 13
$$

$\mathrm{Au}$

$$
\leq 56 \pm 4(1 \gamma, 1 \mathrm{~s})
$$

$<0.0013$

$\pm 0.0001$

$\leq 0.028^{1 \gamma}$ $\pm 0.013$

$<0.0087$ $\pm 0.0016$

Zn

$3.4 \pm 0.1$

$8.8 \pm 0.2$

$3.1 \pm 0.1$

$2.7 \pm 0.1$

$\mathrm{Rb}$

$$
<0.13 \pm 0.03
$$

$<0.30 \pm 0.03$

$0.23^{1 \gamma}$

$\pm 0.06$

$0.12^{1 \gamma}$ $\pm 0.06$

Fe $\quad 31 \pm 2$

$49 \pm 3$

$25 \pm 2$

$28 \pm 2$

$\mathrm{Ca}$

$<920 \pm 170$

$<210 \pm 20$

$<570 \pm 130$

$<330 \pm 60$

La

$0.51 \pm 0.11$

$0.86 \pm 0.07$

$0.53 \pm 0.08$

$0.49 \pm 0.38$ 
Appendix C. continued

\begin{tabular}{lllll}
\hline $\mathrm{W}$ & $0.22 \pm 0.10$ & $0.60 \pm 0.11$ & $0.32 \pm 0.04$ & $0.21 \pm 0.08$ \\
$\mathrm{Na}$ & $16.2 \pm 2.5$ & $150 \pm 240$ & $170 \pm 150$ & $46 \pm 16$ \\
$\mathrm{Ga}$ & $0.61 \pm 0.05$ & $7.6 \pm 1.0(1 \mathrm{~s})$ & $\leq 1.0 \pm 0.31 \gamma$ & $<0.40 \pm 0.10(\mathrm{ls})$ \\
$\mathrm{Br}$ & $<0.21 \pm 0.65$ & $<0.34 \pm 0.04$ & $<0.17 \pm 0.04$ & $<0.27 \pm 0.06$ \\
$\mathrm{As}$ & $<0.50 \pm 0.11$ & $1.2 \pm 0.7(\mathrm{ls})$ & $<0.40 \pm 0.09$ & $<0.65 \pm 0.14$ \\
$\mathrm{Cu}$ & $<15 \pm 3$ & $<26 \pm 1$ & $<570 \pm 130$ & $<18 \pm 4$ \\
$\mathrm{~K}$ & $18 \pm 6$ & $<21 \pm 1$ & $57 \pm 10(2 \mathrm{~s})$ & $20 \pm 6(\mathrm{~s})$ \\
$\mathrm{Ho}$ & $<0.32 \pm 0.07$ & $<2.0 \pm 0.2$ & $<0.26 \pm 0.06$ & $<0.0031 \pm$ \\
& & & & 0.0007 \\
\hline
\end{tabular}

Note: $1 \gamma$ and $2 \gamma$ refer to samples where the impurity level is based on 1 or 2 gamma rays in such a situation that the result is less than certain. Typically this applies to upper limits. The symbol is refers to a positive identification of an impurity in 1 sample ( $2 \mathrm{~s}$ for 2 samples) but not in the other samples of a set, in which case the average is applied to the 1 or 2 samples. Otherwise the impurity limits apply to an average of 3 samples. Table III of Section III.J.3 in the text lists values for the gallium and arsenic levels averaged over the 3 samples. 
Appendix D. Summary of Hazardous Impurities and Radiation Rate Data

\begin{tabular}{|c|c|c|c|c|c|c|}
\hline Element & $\begin{array}{l}\text { Effective }^{1} \sigma \\
\text { Cross } \\
\text { Section } \\
\text { (barns) }\end{array}$ & $A(a m u)$ & $t_{1 / 2}$ (days) & FOM $^{1}$ & $\begin{array}{l}\text { R/hr for } \\
1 \text { Ci@1 } \\
\text { meter } \\
\text { (see 2.) }\end{array}$ & $\begin{array}{l}\text { Shielded } \\
\text { Radiation } \\
\text { Levels } \\
\text { (see 3.) }\end{array}$ \\
\hline $\mathrm{Na}$ & 0.53 & 22.9898 & 0.625 & 0.037 & 1.84 & 0.25 \\
\hline $\mathrm{La}$ & 9.0 & 138.91 & 1.7 & 0.038 & 1.13 & 0.41 \\
\hline$W$ & 10.9 & 183.95 & 0.996 & 0.059 & 0.30 & $1.0(-6)$ \\
\hline $\mathrm{Ga}$ & 1.9 & 69.72 & 0.59 & 0.046 & 1.16 & 0.058 \\
\hline $\mathrm{Sc}$ & 26.5 & 44.956 & 84 & 0.0070 & 1.09 & 0.089 \\
\hline As & 4.3 & 74.9216 & 1.10 & 0.052 & 1.01 & 0.0078 \\
\hline $\mathrm{Br}$ & 1.33 & 159.82 & 1.47 & $5.7(-3)$ & 1.46 & 0.012 \\
\hline $\mathrm{Cu}$ & 3.1 & 63.54 & 0.53 & 0.092 & 0.12 & $2.5(-5)$ \\
\hline Co & 37.0 & 58.933 & 1900 & $3.3(-4)$ & 1.32 & 0.029 \\
\hline $\mathrm{Te}$ & 0.28 & 238.03 & 3.3 & $3.56(-4)$ & 0.22 & $4.5(-4)$ \\
\hline $\mathrm{Ta}$ & 21.0 & 180.948 & 115 & $1.0(-3)$ & 0.68 & 0.026 \\
\hline $\mathrm{Sb}$ & 1.84 & 121.75 & 60 & $2.5(-4)$ & 0.98 & 0.043 \\
\hline $\mathrm{Ba}$ & 0.26 & 238.03 & 12.8 & $8.5(-5)$ & 1.24 & \\
\hline $\mathrm{Ag}$ & 2.2 & 107.87 & 252 & $8.1(-5)$ & 1.43 & 0.073 \\
\hline $\mathrm{Cr}$ & 0.69 & 51.996 & 27.7 & $4.8(-4)$ & 0.016 & \\
\hline Zn & 0.38 & 65.37 & 244 & $2.4(-5)$ & 0.27 & $2.2(-4)$ \\
\hline $\mathrm{Fe}$ & 0.0036 & 55.847 & 45 & $1.4(-6)$ & 0.64 & $3.6(-5)$ \\
\hline
\end{tabular}

Note 1: The effective cross-section is the cross-section for the $(n, \gamma)$ reaction multiplied by the fractional abundance of the target isotope. The FOM (figure of merit) $=\sigma /\left(\mathrm{At}_{1 / 2}\right)$ is a relative measure of the impurity hazard per ppm and is useful for a qualitative understanding of the relative hazards of the primary impurity nuclides produced by reactor irradiation. 
Appendix D con't

Note 2: Radiation levels taken from Ref. 34. These numbers were used in calculations of the radiation levels 2 meters from the shielded source as described in the text (Section IV).

Note 3: Radiation levels in $\mathrm{mrem} / \mathrm{hr}$ after 3 days cool down for $1 \mathrm{ppb}$ level of impurity and on contact with a tungsten shield for the generic GALLEX source design (J. Rich, private communication). These numbers are used in calculations of the radiation levels on contact with the shielded source as described in the text (Section IV). 